\title{
Role of Structural Order and Excess Energy on Ultrafast Free Charge Generation in Hybrid Polythiophene/Si Photovoltaics Probed in Real Time by Near-Infrared Broadband Transient Absorption
}

\author{
Daniel Herrmann, ${ }^{\dagger}$ Sabrina Niesar, ${ }^{\ddagger}$ Christina Scharsich, ${ }^{\S}$ Anna Köhler, ${ }^{\S}$ Martin Stutzmann, ${ }^{\ddagger}$ and \\ Eberhard Riedle ${ }^{*,+}$ \\ ${ }^{\dagger}$ Lehrstuhl für BioMolekulare Optik, Ludwig-Maximilians-Universität München, Oettingenstraße 67, 80538 München, Germany \\ ${ }^{\ddagger}$ Walter Schottky Institut, Technische Universität München, Am Coulombwall 4, 85748 Garching, Germany \\ ${ }^{\S}$ Lehrstuhl EP II, Universität Bayreuth, Universitätsstraße 30, 95440 Bayreuth, Germany
}

Supporting Information

\begin{abstract}
Despite the central role of light absorption and the subsequent generation of free charge carriers in organic and hybrid organic-inorganic photovoltaics, the precise process of this initial photoconversion is still debated. We employ a novel broadband (UV-Vis-NIR) transient absorption spectroscopy setup to probe charge generation and recombination in the thin films of the recently suggested hybrid material combination poly(3-hexylthiophene)/silicon (P3HT/Si) with $40 \mathrm{fs}$ time resolution. Our approach allows for monitoring the

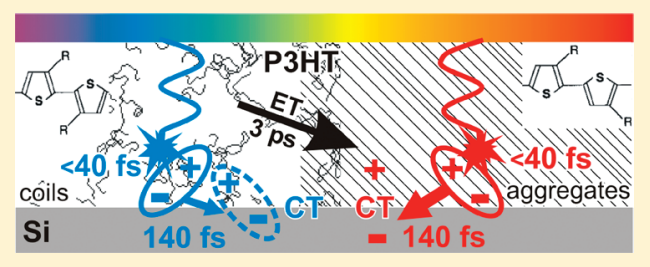
time evolution of the relevant transient species under various excitation intensities and excitation wavelengths. Both in regioregular $(\mathrm{RR})$ and regiorandom $(\mathrm{RRa})$ P3HT, we observe an instant ( $<40 \mathrm{fs}$ ) creation of singlet excitons, which subsequently dissociate to form polarons in $140 \mathrm{fs}$. The quantum yield of polaron formation through dissociation of delocalized excitons is significantly enhanced by adding Si as an electron acceptor, revealing ultrafast electron transfer from P3HT to Si. P3HT/Si films with aggregated RR-P3HT are found to provide free charge carriers in planar as well as in bulk heterojunctions, and losses are due to nongeminate recombination. In contrast for $\mathrm{RRa-P} 3 \mathrm{HT} / \mathrm{Si}$, geminate recombination of bound carriers is observed as the dominant loss mechanism. Site-selective excitation by variation of pump wavelength uncovers an energy transfer from P3HT coils to aggregates with a $1 / e$ transfer time of 3 ps and reveals a factor of 2 more efficient polaron formation using aggregated RR-P3HT compared to disordered RRa-P3HT. Therefore, we find that polymer structural order rather than excess energy is the key criterion for free charge generation in hybrid P3HT/Si solar cells.
\end{abstract}

\section{INTRODUCTION}

Thin film solar cells constitute one of the future technological solutions for sustainable energy supply. A particularly promising route is offered by solar cells made from organic semiconductors or inorganic semiconducting nanoparticles. ${ }^{1-4}$ In recent years, hybrid solar cells based on an organic semiconductor in conjunction with an inorganic nanoscale material are considered as an alternative to purely organic solar cells, as they allow achieving additional functionality by combining the advantages of the two materials. ${ }^{5-9}$ In comparison to polymers, inorganic semiconductors offer a broader spectral range of absorption, particularly in the NIR spectral range, a higher charge carrier mobility, and a better thermal and morphological stability. At the same time, their application in the form of nanoparticles enables the possibility of band gap tuning for sufficiently small nanoparticle diameters and the technological advantages of purely organic solar cells, such as low-cost solution processing, roll-to-roll assembly, or processing onto flexible substrates, are maintained. In the literature, various composites based on $\mathrm{Si}, \mathrm{ZnO}, \mathrm{TiO}_{2}, \mathrm{CdSe}$, and a few other nanomaterials are currently of scientific interest. ${ }^{6-15}$

However, in hybrid and organic solar cells, a detailed fundamental understanding of the processes of light absorption, formation of free polarons, and the subsequent transport of these charges to the electrodes, which are central to their operation, is lacking. In purely organic solar cell devices, these photophysical processes have been shown to depend strongly on the morphology of the heterojunction ${ }^{16}$ so that the device efficiency can be improved significantly by the processing conditions of the film. ${ }^{17-19}$ The widely used and highly attractive polymer poly(3-hexylthiophene) (P3HT) can form two distinct morphological phases associated with different chain conformations. If the P3HT chain adopts a random coil conformation, the resulting film is amorphous. The associated absorption spectrum is unstructured with a maximum centered around $450 \mathrm{~nm}$ (about $2.8 \mathrm{eV})$. This disordered structure prevails for regiorandom P3HT (RRa-P3HT). In regioregular P3HT (RR-P3HT), the polymer chains can planarize and assemble to form weakly coupled $\mathrm{H}$-aggregates, ${ }^{20,21}$ which arrange in closely (a few angstroms) packed two-dimensional lamellar structures via $\pi$-stacking. ${ }^{22-25}$ Their spectroscopic signature is a well-structured absorption spectrum with a $0-0$ vibronic peak around $600 \mathrm{~nm}$ (about $2.0 \mathrm{eV}) .^{21}$

Received: June 1, 2011

Published: September 26, 2011 
Such aggregates are partially formed when RR-P3HT is embedded in a poor solvent or in a film after spin-coating from solution. RRP3HT is a semicrystalline polymer whose degree of crystallization can be controlled by processing conditions. While it became clear that the charge carrier mobility is enhanced in aggregated P3HT chains, ${ }^{26,27}$ studies on the role of the aggregated or coiled conformation in the process of charge carrier generation and separation in organic devices have been emerging only recently. ${ }^{16}$

For the design and operation of a solar cell, it is therefore of crucial importance to understand the influence of morphology on each of the individual photophysical steps. In organic semiconductors, there is widespread agreement about the photoconversion process. The elementary step is light absorption to generate excited states of a donor, followed by diffusion of the excitation to the internal interface formed by a donor adjacent to an acceptor and the decisive electron transfer from the excited donor to the acceptor forming a Coulombically bound electronhole pair. Ideally, this is followed by their dissociation into free charges that move away from the interface, preferentially not suffering bimolecular recombination before being collected at the respective electrodes. Moreover, there is agreement that in purely organic blends the process of charge carrier generation takes place on an ultrafast time scale in the range of $100 \mathrm{fs}^{16,28-31}$

However, the exact mechanism of charge separation is still debated for purely organic solar cells and still in an early phase for hybrid composites. For P3HT in combination with [6,6] phenyl$\mathrm{C}_{61}$-butyric acid methyl ester (PCBM), which has been demonstrated to achieve power conversion efficiencies of around $5 \%,{ }^{32}$ there are suggestions that Frenkel-type excitons are the primary photoexcitations that dissociate into free charges. ${ }^{16,29,30}$ There are indications that the charge separation takes place more efficiently for blends of RR-P3HT:PCBM than for RRa-P3HT: $\mathrm{PCBM}^{16}{ }^{16}$ In contrast, prompt polaron formation during laser excitation was considered for neat P3HT and RRa-P3HT: PCBM $^{30,33}$ For the polymer PCDTBT in combination with PCBM, there are also contradicting interpretations in discussion. On the one hand, it has been suggested that light absorption may directly create mobile electrons and holes by interband $\pi-\pi^{*}$ transitions which would subsequently evolve into Coulombically bound excitons in less than 1 ps. ${ }^{31}$ Similarly, for a composite of a PPV derivative with PCBM, the primary photoexcitation has been suggested to be an ultrafast electron transfer on the time scale of $45 \mathrm{fs} .{ }^{28}$ On the other hand, ultrafast exciton dissociation to form free charges was also considered very recently for PCDTBT:PCBM blends. ${ }^{34}$ For hybrid donor-acceptor materials, detailed ultrafast spectroscopic investigations are still in an early phase. The question, whether light absorption initially creates free charge carriers or excitons, is central to the understanding of light harvesting in organic and hybrid systems. A problem in resolving this issue pertains to the experimental limits of time resolution and spectral range that are accessible to optical probing. Here, we have developed a novel ultrabroadband transient absorption spectroscopy setup with a time resolution of $40 \mathrm{fs}$ covering the entire broad spectral range from 415 to $1150 \mathrm{~nm}$ without interruption. This allows us to monitor both the kinetics of the decay of the primary excitation and its evolution into a charge pair state in thin hybrid films.

In our studies, we focus on composites of $\mathrm{P} 3 \mathrm{HT}$ in combination with silicon which is a particularly promising inorganic acceptor for several reasons. It unifies an almost unlimited abundance with environmentally friendliness, allowing for its widespread use. Silicon additionally provides high electron affinity and allows for rapid electron delocalization and screening after charge transfer which may prevent back transfer and enables fast transport away from the interface. Because of the higher dielectric constant of silicon compared to PCBM, this effect should be even more pronounced as in purely organic films, thus rendering silicon a very promising alternative to PCBM for photovoltaic devices and fundamental studies. Proof-of-principle investigations of charge transfer in hybrid $\mathrm{P} 3 \mathrm{HT} / \mathrm{Si}$ systems were recently performed using electron spin resonance (ESR). ${ }^{9}$ Prototype devices exhibited a relatively high open-circuit voltage of $0.75 \mathrm{~V},{ }^{10}$ and power conversion efficiencies of around $1 \%$ have been achieved. ${ }^{8}$ However, no detailed spectroscopic understanding has been available so far. Because of its current availability in crystalline, nanocrystalline, and amorphous forms, silicon serves as a model system with fundamental implications for various other hybrid or organic material systems. In particular, the exciton dissociation mechanism and therefore the photophysics of charge generation and separation can be studied more clearly in silicon-based devices. The reason for this is that these processes are difficult to assess in the widely studied P3HT/ PCBM composite, since the various PCBM transient signals from the visible to $\mathrm{NIR}^{35}$ superimpose with the transient absorption by the polaron and exciton of P3HT.

In our study, we employ Si nanocrystals (Si-ncs) and polycrystalline silicon (poly-Si) as the electron acceptor in order to study both film geometries of interest, bulk heterojunctions and planar heterojunctions. For the development of efficient commercial solar cells, the bulk heterojunction structure is favored, since it offers a particularly large donor-acceptor interface. The efficiency of planar heterojunctions is limited by the smaller interfacial area, yet the two-dimensional interface area avoids recombination associated with interrupted percolation pathways and cross-currents of electrons and holes. However, as our studies primarily focus on the fundamental principles of the photophysics (charge generation and separation), our devices are optimized for an unambiguous data interpretation. To address the dependence of charge carrier separation on polymer structural order, both disordered RRa-P3HT and semicrystalline RR-P3HT were used.

Our pump-probe setup allows us to directly monitor in real time the process of charge generation in hybrid thin film P3HT/ Si heterojunctions, here at room temperature in the absence of an applied external field. For both RR- and RRa-P3HT, we observe an instant creation of singlet excitons that subsequently dissociate to form polarons on an ultrafast time scale. We observe that the yield of polaron formation through exciton dissociation is significantly enhanced by adding $\mathrm{Si}$ as electron acceptor. Furthermore, we find that the yield of polaron formation and the degree of Coulombic binding of the corresponding polaron pairs formed in $\mathrm{P} 3 \mathrm{HT} / \mathrm{Si}$ depend on the polymer structural order, with efficient free charge carrier generation in RR-P3HT/Si and geminately bound charge carriers formed in RRa-P3HT/Si.

\section{SAMPLE PREPARATION AND SUMMARY OF EX- PERIMENTAL METHODS}

2.1. Sample Preparation. In this work, three different types of poly(3-hexylthiophene) with varying degree of aggregation were used. For fundamental studies of interactions between disordered and ordered regions of P3HT, films of a RR-P3HT (BASF SE, Sepiolid P 100, regioregularity $\left.\sim 95 \%, M_{\mathrm{w}}=50000 \mathrm{~g} / \mathrm{mol}, M_{\mathrm{w}} / M_{\mathrm{n}}=2.2\right)$ were prepared by spin-coating from chloroform $\left(\mathrm{CHCl}_{3}\right)$ solutions with a concentration of $7.5 \mathrm{mg} / \mathrm{mL}$ onto precleaned glass substrates. The same polymer was dissolved in spectroscopically pure chloroform, toluene, and 1,2-dichlorobenzene with concentrations of $0.075 \mathrm{mg} / \mathrm{mL}$ and filled 

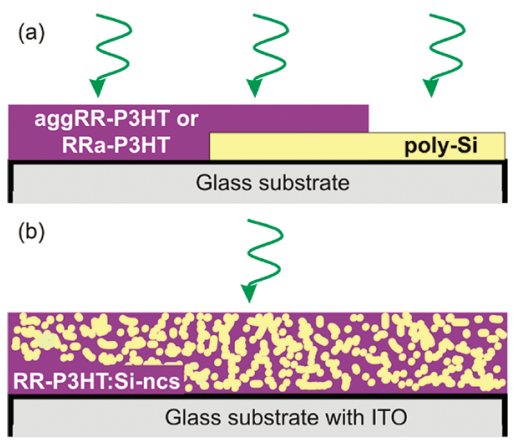

Figure 1. Sample architecture of hybrid P3HT/Si thin film: (a) planar heterojunction and (b) bulk heterojunction on glass substrates.

in $1 \mathrm{~mm}$ fused silica cuvettes for the transient absorption spectroscopy of $\mathrm{P} 3 \mathrm{HT}$ in solution.

$\mathrm{P} 3 \mathrm{HT} / \mathrm{Si}$ thin film heterojunctions with varied morphology were prepared as planar heterojunctions (PHJs, Figure 1a) and bulk heterojunctions (BHJs, Figure $1 \mathrm{~b}$ ). For the $\mathrm{PHJ}$ s with varied polymer structural order, RRa-P3HT (University of Bayreuth, Germany, $M_{w}=40000$ $\left.\mathrm{g} / \mathrm{mol}, M_{\mathrm{w}} / M_{\mathrm{n}}=2.4\right)$ dissolved in chloroform $(5 \mathrm{mg} / \mathrm{mL})$, and RR-P3HT (Rieke Metals, $M_{\mathrm{w}}=39000 \mathrm{~g} / \mathrm{mol}, M_{\mathrm{w}} / M_{\mathrm{n}}=2.0$ ) dissolved in $98 \%$ chloroform and $2 \%$ ethyl acetate (EtAc) $(5 \mathrm{mg} / \mathrm{mL})$ was used. The former was synthesized by treating thiophene with $\mathrm{FeCl}_{3}{ }^{36}$ For the latter, ethyl acetate as nonideal solvent for P3HT was admixed to enhance the aggregation of the RR-P3HT molecules, ${ }^{37-39}$ which is therefore referred to as aggRR-P3HT. All sample preparations were performed under argon atmosphere.

Silicon nanocrystals (Si-ncs) were synthesized in a low-pressure microwave plasma reactor by decomposition of silane. ${ }^{40}$ The pressure of the process gases and the microwave power determine the mean diameter of the Si-ncs. Phosphorus doping of the Si-ncs was achieved by adding phosphine during growth and increases the carrier mobility in the Si-ncs. The nominal doping concentration is defined by the phosphine flow and the flow of the total precursor gas. The Si-ncs used in this work have a mean diameter of 4 and $18 \mathrm{~nm}$ and nominal doping concentrations of $5 \times 10^{20}$ and $6.5 \times 10^{19} \mathrm{~cm}^{-3}$, respectively. The standard deviation of the particle diameter is typically $\sigma \approx 1.4 \mathrm{~nm}$.

Blend films of RR-P3HT and Si-ncs with a weight ratio of 5:1 were spin-coated under nitrogen atmosphere from solutions in chloroform (concentration $7.5 \mathrm{mg} / \mathrm{mL}$ ) to form RR-P3HT:Si-ncs BHJs (Figure 1b). For the intended application as a solar cell, $15 \mathrm{~mm} \times 15 \mathrm{~mm} \times 1.1 \mathrm{~mm}$ aluminoborosilicate with an approximately $110 \mathrm{~nm}$ thick conductive indium tin oxide layer (ITO, Delta Technologies, $R_{\mathrm{s}}=5-15 \Omega$ ) was used as a substrate. Before spin-coating, the substrates were cleaned by subsequent ultrasonic treatment in acetone and isopropanol for $10 \mathrm{~min}$ each. A typical sample layer thickness of $100 \mathrm{~nm}$ was achieved. The thin film samples were sealed against air by using fused silica coverslips (150 $\mu \mathrm{m}$ thin) and silicone sealant.

The refractive index of the Si-ncs was previously measured to be about 2.0 with only a slight monotonic decrease with wavelength. The refractive index of the blend films can be expected to be close to the one of a neat P3HT film, which has a reported index of around 1.7-2.0. ${ }^{41}$ Consequently no significant change of the Fresnel losses upon mixing of the two materials is expected and the weak excitation should also not lead to a transient change.

Polycrystalline silicon (poly-Si) films were prepared by silver-induced layer exchange (AgILE). ${ }^{42}$ For a resulting poly-Si film thickness of $30 \mathrm{~nm}$, an amorphous silicon precursor layer $(50 \mathrm{~nm})$ was grown on top of a $30 \mathrm{~nm}$ silver layer on a fused silica substrate. The crystallization was performed at $800{ }^{\circ} \mathrm{C}$ for $10 \mathrm{~h}$ under nitrogen atmosphere. Afterwards, the
Table 1. List of Samples Used in This Work along with Their Abbreviations

\begin{tabular}{ll}
\multicolumn{1}{c}{ sample } & \multicolumn{1}{c}{ abbreviation } \\
RR-P3HT (BASF), spun from $\mathrm{CHCl}_{3}$ & RR-P3HT \\
RRa-P3HT (Bayreuth), spun from $\mathrm{CHCl}_{3}$ & RRa-P3HT \\
RR-P3HT (Rieke), spun from $\mathrm{CHCl}_{3} / \mathrm{EtAc}$ & aggRR-P3HT \\
silicon nanocrystals & Si-ncs \\
polycrystalline silicon & poly-Si \\
RR-P3HT:Si-ncs bulk heterojunction & RR-P3HT:Si-ncs BHJ \\
RRa-P3HT/poly-Si planar heterojunction & RRa-P3HT/poly-Si PHJ \\
aggRR-P3HT/poly-Si planar heterojunction & aggRR-P3HT/poly-Si PHJ \\
\hline
\end{tabular}

silver was etched away with a 1:1 mixture of hydrogen peroxide and ammonia solution at $100^{\circ} \mathrm{C}$. We prepared PHJs of $40 \mathrm{~nm}$ RRa-P3HT or aggRR-P3HT and $30 \mathrm{~nm}$ poly-Si (aggRR-P3HT/poly-Si PHJ and RRa$\mathrm{P} 3 \mathrm{HT} /$ poly-Si PHJ, Figure 1b) under nitrogen atmosphere. To be able to perform TA spectroscopy of the individual materials and of the heterojunctions, the poly-Si covers only a part of the substrate (Figure 1a). Furthermore, we removed a stripe of the P3HT on the Si side using a cotton tip with chloroform. A summary of the sample structures used is given in Table 1.

2.2. Summary of Experimental Methods. Details about the basic optical and morphologic characterization of the thin film samples and the novel ultrafast transient absorption spectrometer are given in the Supporting Information. To investigate the nature of the photoexcitations and their inherent kinetics after visible excitation, we used an ultrafast $1 \mathrm{kHz}$ pump-probe setup with a probe range of $290-740 \mathrm{~nm} .{ }^{43}$ The visible excitation with $15 \mathrm{fs}$ pulses in the range from 450 to $720 \mathrm{~nm}$ is accomplished by a noncollinear optical parametric amplifier (NOPA). ${ }^{44,45}$ We expand the probe range to $415-1150 \mathrm{~nm}$ by the combination of two supercontinuum generation stages and a NIR-OPA operated at $1180 \mathrm{~nm}$, allowing for broadband UV-Vis-NIR TA spectroscopy with $40 \mathrm{fs}$ time resolution (Figure S3). This OPA was seeded with a supercontinuum from a YAG crystal ${ }^{46}$ and generated the Vis-NIR probe continuum in a $\mathrm{CaF}_{2}$ crystal. The pump and probe were focused towards the sample to a 210 and $110 \mu \mathrm{m} 1 / e^{2}$ beam diameter that allows ensemble averaging over the finely grained morphology of the thin films to mimic their usage as photovoltaic device and to ensure low local excitation densities.

\section{RESULTS AND DISCUSSION}

3.1. Structural Implications of Regioregularity and Solvent. In our study, we aim to understand the process of charge carrier generation in the polymer $\mathrm{P} 3 \mathrm{HT}$ and in the hybrid system formed by $\mathrm{P} 3 \mathrm{HT}$ in combination with silicon. $\mathrm{P} 3 \mathrm{HT}$ is a semicrystalline polymer whose degree of aggregation depends on the degree of regioregularity of the chain as well as on the solvent used. In order to assess the role of aggregated P3HT chains in the charge generation process, we employed samples with different degrees of regioregularity and spun from different solvents, as summarized in Table 1 . The resulting structure of the films was carefully monitored via atomic force microscopy (AFM). The corresponding topographical images in $2 \mathrm{D}$ and 3D plots are shown in Figures $2 \mathrm{a}-\mathrm{c}$ and Figure $\mathrm{S} 1$ and reveal a varied aggregation: While RRa-P3HT exhibits a rather long spatial coherence length (Figure 2a, Figure S1a), aggRR-P3HT possesses a fine structure (Figure 2c, Figure S1b). RR-P3HT is between these two extremes (Figure 2b).

The differences can be understood by regarding the different processing conditions. In the case of aggRR-P3HT, we have used chloroform as the main solvent, which exhibits a lower $\left(61^{\circ} \mathrm{C}\right)$ 


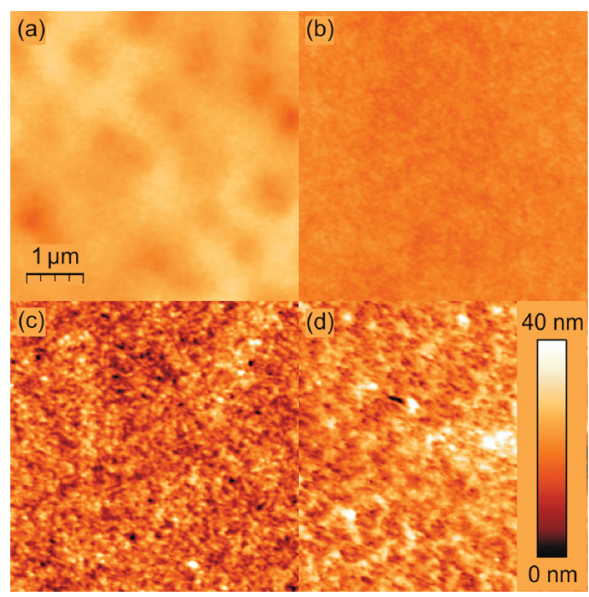

Figure 2. Topographical AFM images of spin-coated films of (a) RRaP3HT (rms roughness $R_{\mathrm{q}}=2.2 \mathrm{~nm}$ ), (b) RR-P3HT $\left(R_{\mathrm{q}}=1.2 \mathrm{~nm}\right)$, (c) aggRR-P3HT $\left(R_{\mathrm{q}}=5.4 \mathrm{~nm}\right)$ directly on glass substrate as well as $(\mathrm{d})$ aggRR-P3HT on top of a $30 \mathrm{~nm}$ thin polycrystalline Si layer $\left(R_{\mathrm{q}}=\right.$ $6.3 \mathrm{~nm}$ ). For (c) and (d), 2\% ethyl acetate was mixed with the chloroform solution to enhance the aggregation of the RR-P3HT molecules.

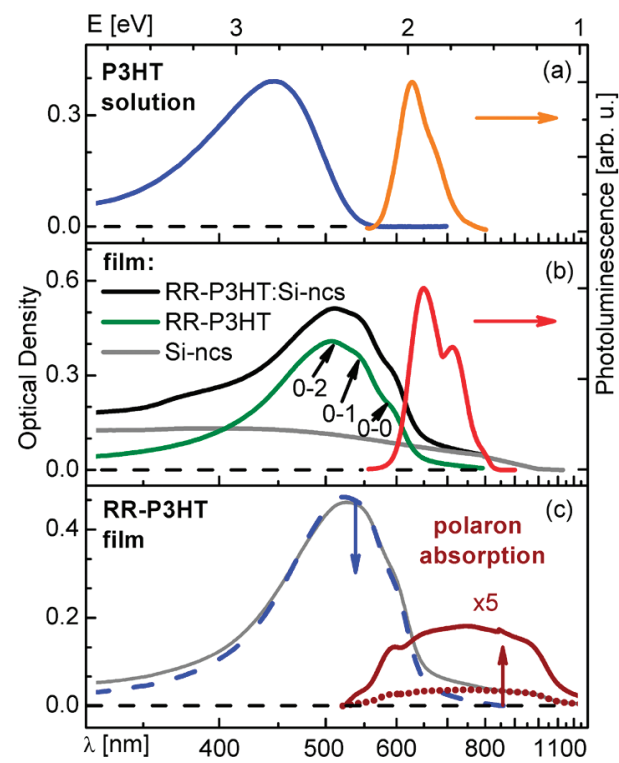

Figure 3. Absorbance and photoluminescence spectra of P3HT in (a) dilute chloroform solution and (b) of RR-P3HT film. The absorbances of Si-ncs and RR-P3HT:Si-ncs (5:1) BHJ as film are also shown. (c) Absorbance spectra of RR-P3HT (blue dashes), oxidized RR-P3HT film after adding $\mathrm{FeCl}_{3}$ (solid gray curve) and $\mathrm{P} 3 \mathrm{HT}$ film polaron absorption (brown dots and brown solid curve).

boiling point as the nonideal solvent ethyl acetate $\left(77^{\circ} \mathrm{C}\right)$. The already partially aggregated polymer falls out of solution before the main solvent is fully dissipated and before the film has fully dried, leading to enhanced aggregation evident in the fine structure of the AFM data. Comparison of Figure $2 \mathrm{c}$ and Figure $2 \mathrm{~d}$ further reveals that when spin-coating aggRR-P3HT directly on poly-Si instead of glass, the fine structure indicating the aggregation is maintained.

3.2. Optical Characterization. The amount of aggregation present in a P3HT sample manifests itself not only in the AFM

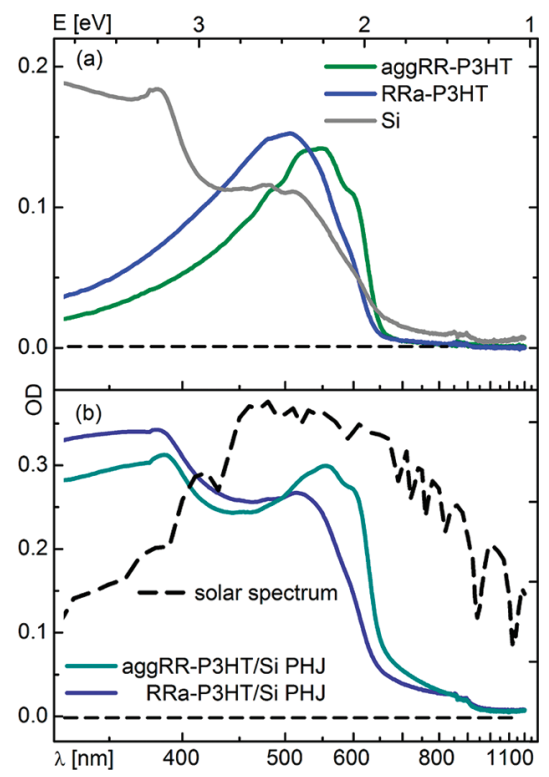

Figure 4. (a) Absorbance spectra of neat RRa-P3HT, RR-P3HT + ethyl acetate (aggRR-P3HT), and neat poly-Si and (b) the corresponding $\mathrm{P} 3 \mathrm{HT} /$ poly-Si PHJs samples compared with the solar spectrum.

characteristics but also in the optical spectra. Figure 3 shows the absorbance (OD) and photoluminescence (PL) spectra of RR$\mathrm{P} 3 \mathrm{HT}$ in the dilute chloroform solution and for thin film samples used in this work. In dilute solution with a good solvent such as chloroform, RR-P3HT is known to adopt a random coil conformation with a distribution of short conjugation lengths. This results in a structureless absorbance that has its maximum at $446 \mathrm{~nm}$ and in a red-shifted more structured PL with peaks at 627 and $688 \mathrm{~nm}$ (Figure 3a). The PL originates from the longest polymer segments after relaxation of the initial photoexcitation. In film, the RR-P3HT chains can planarize to form weakly interacting $\mathrm{H}$-aggregates that are embedded in a matrix of amorphous coiled P3HT chains. The resulting absorption thus consists of a superposition of absorption by coiled chains and absorption by planar, highly conjugated and aggregated chains. ${ }^{21}$ Consequently, the absorption is shifted to longer wavelengths, is broadened, and shows vibronic peaks at 518, 558, and $608 \mathrm{~nm}$ (Figure $3 \mathrm{~b}$ ) due to the $0-2,0-1$, and $0-0$ transitions, respectively, in agreement with literature data. ${ }^{21}$ The corresponding PL also contains vibronic structure with peaks at 650,712 , and $800 \mathrm{~nm}$ (mainly the $\mathrm{C}=\mathrm{C}$ symmetric stretching mode, $1452 \mathrm{~cm}^{-1}$ ).

By considering the intensity of absorption between 400 and $500 \mathrm{~nm}$ and by considering the vibrational structure around $600 \mathrm{~nm}$ through a modified Franck-Condon analysis as described in refs 21 and 47, it is possible to derive the amount of aggregates present in a film (Figure S2). Figure 4a shows the absorption spectra of the two limiting cases given in our study, that is, RRa-P3HT and aggRR-P3HT. The absorption of poly-Si is also shown for comparison. Analyzing the P3HT absorption in this fashion, with the assumption that the absorption coefficient of aggregated chains is 1.39 times that of coiled chains, ${ }^{47}$ yields a percentage of $(38 \pm 5) \%$ aggregates for the aggRR-P3HT sample and of $(24 \pm 5) \%$ aggregates for the RRa-P3HT sample (Figure S2). RRa-P3HT possesses less aggregation and a reduced conjugation length compared to RR-P3HT, which was attributed to the adverse steric repulsive interactions between the hexyl side chains and the sulfur. ${ }^{17,48}$ Figures $3 \mathrm{~b}$ and $4 \mathrm{~b}$ illustrate that when 
the P3HT is intermixed with Si-ncs or spun on top of poly-Si, the absorption spectrum is given by a superposition of the individual components. Thus, in agreement with the AFM data, spinning a film on top of a silicon substrate does not seem to affect the amount of aggregates formed. Figure $4 \mathrm{~b}$ further demonstrates the good match of the heterojunction absorption against the solar irradiation spectrum that is essential for efficient solar cells.

In order to study the process of charge generation in these material systems, we do not only need to know how many aggregated or coiled chains are present in the P3HT but also require a spectroscopic signature for charges in $\mathrm{P} 3 \mathrm{HT}$ films. P3HT thin films can be chemically oxidized employing a strong oxidant. ${ }^{25,49}$ Figure $3 \mathrm{c}$ shows the absorbance spectrum of thus oxidized RRP3HT thin films after dipping into $20 \mathrm{ppm}$ solution of iron(III) chloride $\left(\mathrm{FeCl}_{3}\right)$ in acetonitrile $\left(\mathrm{CH}_{3} \mathrm{CN}\right)$ for 1 min followed by rinsing with acetonitrile to remove excess oxidant. From the raw spectrum of the treated film we obtain the $\mathrm{P} 3 \mathrm{HT}$ polaron $\left(\mathrm{P}_{3} \mathrm{HT}^{+}\right)$absorption as follows. The treatment with $\mathrm{FeCl}_{3}$ decreases the known absorbance of the neutral P3HT molecule (blue dashes) and increases absorbance in the range from about 560 to $1150 \mathrm{~nm}$ (brown dots). Partial saturation occurs at treatment longer than $2 \mathrm{~min}$. The difference between the blue dashed line scaled to the peak of the gray solid curve in Figure $3 \mathrm{c}$ and the gray solid curve reveals the RR-P3HT film polaron absorption (brown dots and brown solid line). It ranges from about 560 to $1150 \mathrm{~nm}$ with a characteristic shape and increases again up to the mid-infrared spectral region. The same oxidation was performed for RRa- and aggRR-P3HT, and the results are shown in Figure 5. Comparable but, because of reduced conjugation length, slightly shifted results of the cation absorption are obtained for P3HT in solution where chemical oxidation was introduced via adding pentachloroantimonate (Figure S6, see details in Supporting Information). Similar oxidation experiments as the ones presented here have been reported and yielded very similar spectra. ${ }^{25,49,50}$

The polaron absorption spectrum, that we obtain in the P3HT films (Figure 3c), closely matches the subgap polaron absorption bands obtained by CW photoinduced absorption (PA) measurements as reported in recent publications. ${ }^{71,25,51,52}$ Therefore, we use our measured polaron absorption spectra for the individual P3HT types for comparison with the transient absorption spectra throughout the whole work.

3.3. Primary Photoexcitations. The thoroughly characterized samples and the systematic variation of their composition allow the study of the excitations formed after illumination. To directly probe the nature of the primary photoexcitations and the inherent photovoltaic conversion processes in hybrid $\mathrm{P} 3 \mathrm{HT} / \mathrm{Si}$ layers, we performed ultrafast pump-probe (transient absorption, TA) spectroscopy. The thin film samples were designed to enable direct comparison between neat P3HT, neat $\mathrm{Si}$, and the $\mathrm{P} 3 \mathrm{HT} / \mathrm{Si}$ heterojunction in ultrafast TA spectroscopy at the same experimental conditions by moving the relevant sample regions into the pump-probe region. We chose pump and probe beam diameters at the sample that allow for ensemble averaging over the finely grained morphology of the thin films to mimic their usage as photovoltaic device and to ensure low excitation fluence $\left(9 \mu \mathrm{J} / \mathrm{cm}^{2}\right.$ at $\left.518 \mathrm{~nm}\right)$ comparable to the solar exposure. The low excitation fluence was also chosen to prevent modulation of the transient signatures due to a thermally induced spectral blue shift, which might occur at high excitation fluences. ${ }^{53}$

Figure 5 shows typical Vis-NIR TA spectra for aggP3HT/ poly-Si PHJ, RRa-P3HT/poly-Si PHJ, and RR-P3HT:Si-ncs BHJ (red solid curves) compared with the TA spectra of the corresponding neat polymer film (black solid curves). Spectra were recorded for the full range from 10 ps prior to the pump pulse up to $2 \mathrm{~ns}$ after the pump pulse (see Figure S11). Spectra taken at $300 \mathrm{fs}, 20 \mathrm{ps}$, and 1-2 ns pump-probe delay are shown. The spectra are composed of negative $\triangle \mathrm{OD}$ signals in the spectral region of the P3HT film absorption between 415 and $630 \mathrm{~nm}$ because of ground state bleach (GSB) and positive signals at longer wavelengths due to photoinduced absorption (PIA), i.e. excitons and polarons.

In order to directly compare the transient spectra and the kinetics of the charged species of the various neat $\mathrm{P} 3 \mathrm{HT}$ with the corresponding $\mathrm{P} 3 \mathrm{HT} / \mathrm{Si}$ heterojunctions, the same number of initial photoexcitations needs to be considered. Small deviations in this number of initial photoexcitations occur in the experiment due to small variations in film quality, film thickness, and thus absorption or due to small changes in pump beam size and pump energy between the various samples. The integral over the GSB area equals the product of the excitation density times the strength of the first electronic transition of $\mathrm{P} 3 \mathrm{HT}$ regardless of small variations of Franck-Condon activity due to differing morphologies of $\mathrm{P} 3 \mathrm{HT}$ in the various samples. ${ }^{54}$ The integral therefore represents a good relative measure of the number of initial photoexcitations. We use the transient spectrum of the aggRR-P3HT film as reference. The TA spectra of the other polymers and $\mathrm{PHJs}$ are each scaled by a small factor $(0.7-1.3)$ for the whole data set of 250 time steps and 512 wavelengths. As a result, all spectra yield the same GSB area integral at the earliest usable time delay. We found that any delay time between 0 and 80 fs gives the same result. This procedure allows for considering the same number of initial photoexcitations in the films. Any changes in the optical density ( $\triangle \mathrm{OD}$ in \%) that would be seen under reference conditions are then solely inherent to the different nature of the sample.

For each measurement, the pairwise difference between TA spectra of the heterojunction and the neat polymer is calculated (green solid curves). For comparison, the particular P3HT film polaron absorption spectrum (blue dashes) is included. The GSB of aggRR-P3HT and RR-P3HT clearly reveals the characteristic vibronic structure of the $\mathrm{P} 3 \mathrm{HT}$ aggregate, in particular at 1-2 ns after excitation, which does not show a spectral shift during the delay times covered in the TA measurements. In Figure 5, we further observe the relative signal ratio between the $0-0$ and $0-1$ GSB peak to change over time and, most importantly, the GSB at $500 \mathrm{~nm}$ to reduce with time. As detailed further below in section 3.6.1 and in the Supporting Information, this is a signature that energy transfer takes place from coiled chains to aggregated chains.

Since the GSB is due to those P3HT chains which are not in the electronic ground state, it directly monitors the total number of photoexcitations such as excitons or polarons still present at a given delay time. The broadband PIA signals with positive $\triangle \mathrm{OD}$ (compare Figure 5) are adjacent to the GSB and exceed the NIR detection range. We compare the PIA for the hybrid heterojunctions (red solid curves) to the corresponding neat polymer (black solid curves) and the difference spectra for each pair with the chemically obtained P3HT film polaron absorption spectrum. We find that the difference spectra match the P3HT film polaron absorption spectra in the range of about $620-850 \mathrm{~nm}$. The comparison also reveals a region of enhanced PIA through adding Si to P3HT. From this we conclude that combining P3HT with silicon results in the generation of 

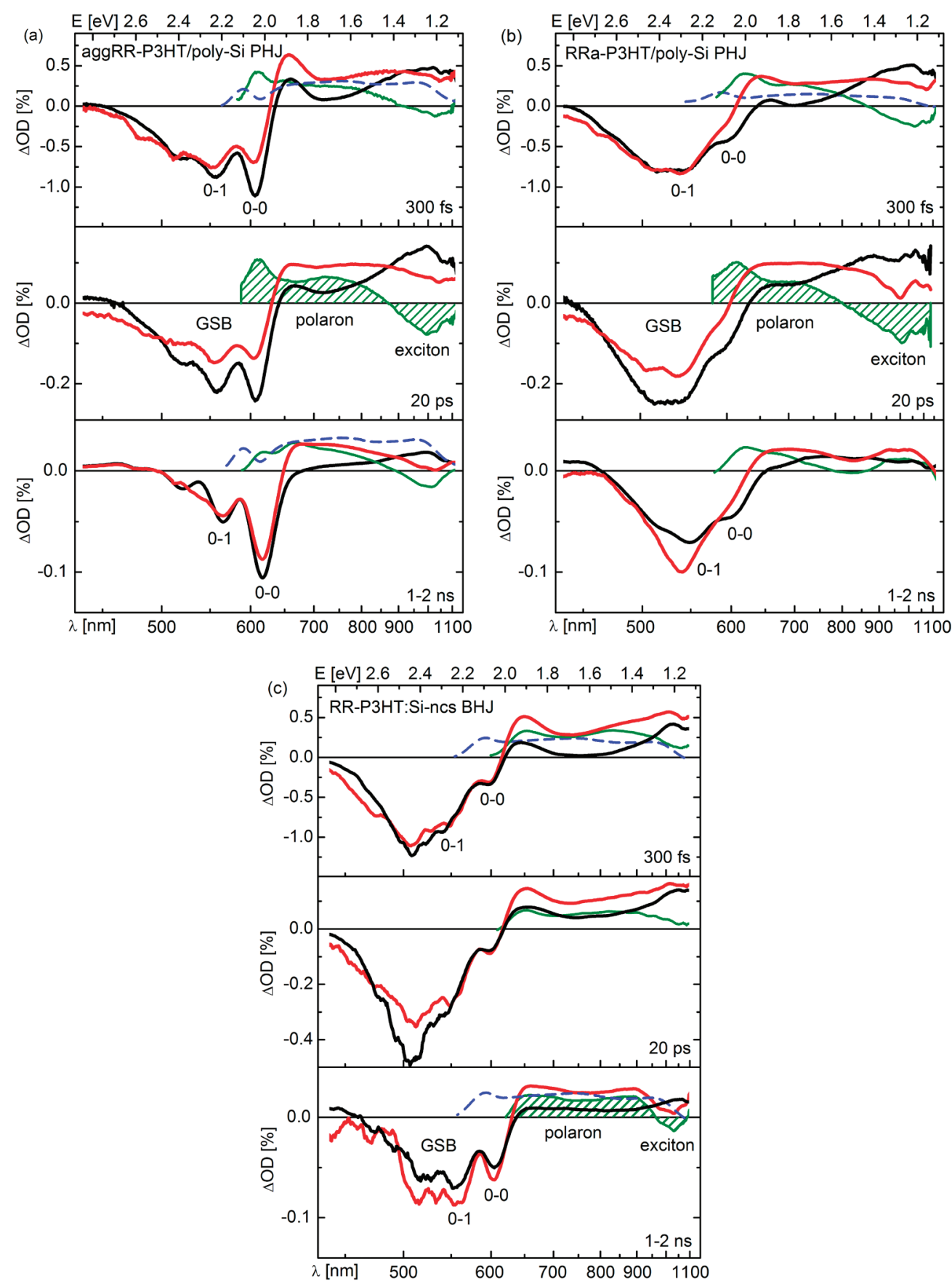

Figure 5. Transient absorption of (a) aggRR-P3HT/poly-Si PHJ, (b) RRa-P3HT/poly-Si PHJ and (c) RR-P3HT:Si-ncs BHJ for 300 fs, 20 ps and $1-2 \mathrm{~ns}$ pump - probe delay. The spectra are scaled to the same number of initial photoexcitations. The P3HT polaron absorption (blue dashes) and the difference (green solid curve) between neat P3HT (black solid curve) and P3HT/Si (red solid curve) are compared. Excitation: $518 \mathrm{~nm}$, $9 \mu \mathrm{J} / \mathrm{cm}^{2}$.

additional P3HT cations, i.e. polarons. In the neat P3HT film, this polaron absorption also seems to be present, albeit at a significantly reduced level. From a comparison of the signal magnitude at $660 \mathrm{~nm}$ in the neat P3HT film and in the hybrid heterojunction we infer that the polaron yield in the $\mathrm{P} 3 \mathrm{HT} / \mathrm{Si}$ heterojunctions is more than a factor of 2 higher. Clearly, there must be ultrafast electron transfer from $\mathrm{P} 3 \mathrm{HT}$ to $\mathrm{Si}$ in $\mathrm{PHJ}$ and $\mathrm{BHJ}$ morphologies. Consequently, $\mathrm{Si}$ is a promising electron acceptor for hybrid photovoltaic devices, in accordance with results by LESR. ${ }^{9}$
We now focus on the PIA spectra in the range of $900-1100 \mathrm{~nm}$. In this range, the difference spectrum, given by the green solid curve in Figure 5, does not match the polaron absorption spectrum (blue dots). In agreement with previous investigations, ${ }^{16,25,29,30}$ we assign the photoinduced absorption in this spectral range to absorption by singlet excitons. To support this assignment, we performed TA measurements of RR-P3HT in various dilute solutions, where the intermolecular distance is high. We found that under these conditions, P3HT cation formation does not take place (Figure S7, see details in Supporting Information). 

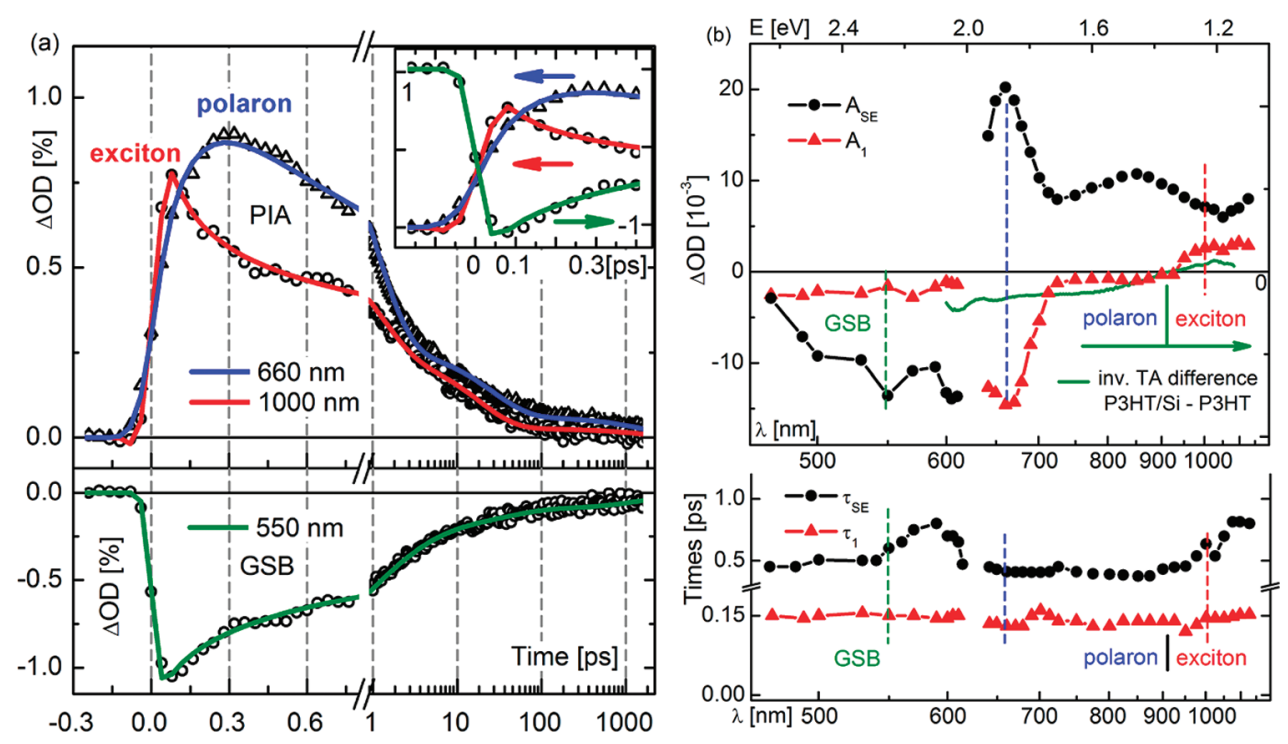

Figure 6. (a) Experimental TA signals (open symbols) as function of the pump-probe delay with corresponding fits (solid curves) of GSB (green), singlet exciton (red), and polaron (blue) for the aggRR-P3HT/poly-Si PHJ. The fit parameters are listed in Table 2. (b) PIA band single-channel fit amplitudes and time constants for the $140 \mathrm{fs}$ component (red triangles) and the $1 \mathrm{ps}$ component (black dots). The dashed lines are the signals taken for (a). The band assignment (inverted green curve) from Figure 5a agrees with the separation of polaron and exciton signatures via the fit amplitudes of the 140 fs component.

The singlet exciton leads to stimulated emission and undergoes intersystem crossing on the nanosecond time scale to a triplet state with a characteristic transition at $1.49 \mathrm{eV}(830 \mathrm{~nm})$. The energetic position of the $\mathrm{P} 3 \mathrm{HT}$ triplet transition confirms and adds to recent experimental and theoretical investigations. ${ }^{16,55}$ In addition to confirming the assignment of the singlet and triplet excitons, this measurement also implies that excitation dissociation does not occur efficiently on a single polymer chain.

Moreover, the difference spectrum between the hybrid system and the neat P3HT in Figure 5 shows a reduced PIA in the range of 900-1150 nm, which suggests that adding silicon to P3HT rapidly reduces the number of singlet excitons. The time evolution of the TA signals will be analyzed in detail further below. No transient signatures of stimulated emission (SE) or a triplet state are detected in any of the film measurements. This observation and the concomitant increase of polaron absorption and reduction of exciton absorption when P3HT is combined with silicon suggest the dissociation of singlet excitons as a path for polaron formation. We therefore conclude that the enhancement of polaron yield in P3HT by adding Si is due to an ultrafast electron transfer from P3HT (electron donor) to $\mathrm{Si}$ (electron acceptor) for all morphologies, namely $\mathrm{PHJ}$ and $\mathrm{BHJ}$. We note that no transient signatures could be detected for the neat Si films or for excitation of the $\mathrm{P} 3 \mathrm{HT} / \mathrm{Si}$ heterojunctions with a pump wavelength of $720 \mathrm{~nm}$, which is outside the P3HT absorption (Figures $3 \mathrm{~b}$ and $4 \mathrm{a}$ ). This shows that the observed effect of enhanced polaron absorption and reduced exciton absorption is due to electron transfer from excited P3HT to Si and not due to optical effects such as a transient change of index of refraction in Si.

So far we have assigned the various features of the TA spectra to photoexcitations, and the general observations pertained to all sample structures. We now consider the quantitative differences that arise between the various samples. In agreement with previous work, ${ }^{16,29,33}$ we find some polaron formation to occur also for the neat P3HT films. Taking the signal magnitude around $660 \mathrm{~nm}$ as a measure for the amount of polaron formation, we find the initial yield to decrease in the order aggRR-P3HT, RR-P3HT, and RRa-P3HT. This is also the order in which the amount of aggregated chains in the film decreases (Figure 2). Therefore, the obtained clear discrimination between the TA spectra of the three types of P3HT matches the distinct differences in the corresponding optical and structural properties. We thus associate a higher polaron yield with an enhanced degree of aggregation and extended conjugation, enabling highly delocalized excitations and charge carriers with high mobility. ${ }^{24,25}$

This effect of the P3HT structural order on the polaron yield is also manifested in the hybrid heterojunctions. Comparison of the polaron absorption signal (at $660 \mathrm{~nm}$; see red curve in Figure 5) indicates about a factor of 2 more efficient initial polaron formation for aggRR-P3HT/poly-Si PHJ compared to RRa$\mathrm{P} 3 \mathrm{HT} /$ poly-Si PHJ. We attribute this finding to the higher degree of conjugation, pronounced exciton delocalization, and an increased mobility of charge carriers which enable a more efficient charge transfer. A possibly more favorable free energy of charge generation through improved band alignment for ordered P3HT chains remains to be investigated. ${ }^{56}$ Recent results state a rise only of the HOMO energy level and no change of the LUMO level through chain ordering. ${ }^{57}$ For a possible photovoltaic application, we compared RR-P3HT:Si-ncs BHJs with aggRR$\mathrm{P} 3 \mathrm{HT} /$ poly-Si PHJs. The polaron yield is higher and their lifetime is slightly longer in aggRR-P3HT/poly-Si PHJ than in RR-P3HT:Si-ncs BHJ as becomes evident through comparison of the TA spectra in Figure 5a,c. The former can be attributed to the intermediate $\mathrm{P} 3 \mathrm{HT}$ type used in the $\mathrm{BHJ}$. The latter is assigned to $\mathrm{Si}$ dangling bond defects in Si-ncs which act as recombination centers. To address this, we are currently working on postgrowth treatments, e.g., HF etching, vacuum annealing, and surface functionalization, which improve the surface and defect properties. ${ }^{58}$ Moreover, interrupted percolation paths in the current $\mathrm{BHJ}$ morphology can also lead to enhanced recombination and limit the efficiency, as not the entire amount of photoinduced charge carriers can move to the electrodes. To overcome this, the fabrication of a defined $\mathrm{BHJ}$ morphology via nanoimprinting is a promising approach. 
In summary, our TA measurements point out that polymer structural order plays a significant role in hybrid solar cells, though Si can significantly enhance the initial polaron yield even in the disordered RRa-P3HT (Figure 5b). Below, it will be investigated whether bound polaron pairs or mobile charge carriers are formed in the $\mathrm{P} 3 \mathrm{HT} / \mathrm{Si}$ heterojunctions.

3.4. Temporal Evolution of the Charge Generation Process. Having identified the features of GSB (about 415-620 nm), polaron absorption (about 620-900 nm), and singlet-exciton absorption (900-1150 nm), we now consider their inherent kinetics in a quantitative fashion. Figure 6a shows the evolution of the TA signals for the GSB (at $550 \mathrm{~nm}$ ), the polaron absorption (at $660 \mathrm{~nm}$ ), and the singlet exciton absorption (at $1000 \mathrm{~nm}$ ) of aggRR-P3HT/poly-Si PHJ on a time scale up to 2 ns with a time resolution of 40 fs. From Figure $6 \mathrm{a}$, a few observations can be made immediately. First, the GSB and the singlet exciton absorption reach their maximum signal with a rise time of $40 \mathrm{fs}$ (see also the inset), i.e., within the experimental resolution. This observation is important, as it implies that the exciton is formed directly upon photoexcitation. Second, the polaron absorption signal shows a delayed rise. It reaches its maximum at about $300 \mathrm{fs}$. This rise of the polaron signal is matched by a corresponding initial decay of the singlet exciton absorption signal. This suggests that the singlet exciton, formed by absorption, decays to form polarons. Third, the ultrafast time evolution of the GSB and the singlet exciton absorption signal is very similar. This can be readily understood by an additional ultrafast nonradiative decay mechanism of at least some of the excited P3HT molecules. A minimal rate model that allows relaxation of the exciton into the polaron by dissociation and to the P3HT ground state by nonradiative decay directly renders the result that the yield of each channel is given by the ratio of the individual rate to the sum of both. ${ }^{59}$ The spectroscopic signal is additionally weighted by the respective extinction coefficients. The exciton serves as a reservoir, and the same femtosecond kinetics is observed for the decay of the exciton signal and the recovery of the GSB. ${ }^{59}$ Nonradiative electronic decay on the femtosecond time scale is now widely reported for a large variety of molecular systems ${ }^{60-64}$ and believed to be frequently mediated by conical intersections. ${ }^{65,66}$ Whether a conical intersection is also responsible for the observed ultrafast nonradiative decay in P3HT films has to be clarified in the future. It has recently been established in conjugated polymers that exciton localization occurs in tens of femtoseconds and leads to nonemissive states. ${ }^{67,68}$ Single-molecule spectroscopy has correlated the ultrafast relaxation to aggregated regions of the polymer. ${ }^{69}$

To further substantiate the conclusion that the polarons are formed from the excitons, we have fitted the decay of the transient data. Already a visual interpretation of the kinetic traces shows that there is the ultrafast signal change as discussed above and an additional slower component. It is possible to model the decay of the GSB and the singlet exciton absorption as a stretched exponential (SE) curve. A stretched exponential decay is expected for films that possess an ensemble of ordered and disordered regions and a correspondingly broad distribution of decay times. ${ }^{70,71}$ As we are particularly interested in the initial signal changes, we treat the ultrafast component separately by fitting the GSB and the singlet exciton absorption according to the function

$$
\Delta O D=A_{1} \exp \left(-t / \tau_{1}\right)+A_{\mathrm{SE}} \exp \left(-t / \tau_{\mathrm{SE}}\right)^{\beta}+\text { const }
$$

We also use eq 1 to fit the polaron absorption signal. The polaron decay will also be characterized by a distribution of
Table 2. Fit Parameters: Amplitudes and Time Constants for the Transient Species in Figure 6

\begin{tabular}{llcl} 
parameter & polaron $(660 \mathrm{~nm})$ & exciton $(1000 \mathrm{~nm})$ & GSB $(550 \mathrm{~nm})$ \\
$A_{1}$ & $-14.6 \times 10^{-3}$ & $2.6 \times 10^{-3}$ & $-1.6 \times 10^{-3}$ \\
$\tau_{1}(\mathrm{ps})$ & 0.14 & 0.14 & 0.14 \\
$A_{\mathrm{SE}}$ & $20.2 \times 10^{-3}$ & $7.1 \times 10^{-3}$ & $-13.6 \times 10^{-3}$ \\
$\tau_{\mathrm{SE}}(\mathrm{ps})$ & 0.41 & 0.63 & 0.6 \\
\hline
\end{tabular}

relaxation times, because of various on-chain and interchain recombination paths. Therefore, the use of a stretched exponential fit for the decay kinetics is justified. ${ }^{71}$ The fit curves obtained are indicated as solid colored curves in Figure 6a. The fit parameters $A_{1}, A_{\mathrm{SE}}, \tau_{1}, \tau_{\mathrm{SE}}$ are listed in Table 2 for the $\Delta \mathrm{OD}$ signals at $660 \mathrm{~nm}$ (polaron), $1000 \mathrm{~nm}$ (exciton), and $550 \mathrm{~nm}$ (GSB). The exponent $\beta$ was found to be 0.5 in all cases. Figure $6 \mathrm{~b}$ illustrates how these fit parameters vary as a function of probe wavelength across the entire detected spectral range.

We find that the singlet exciton absorption and the GSB both decay with a similar first ultrafast time constant of $140 \mathrm{fs}$, followed by a slower decay, for which the combination of $\tau_{\mathrm{SE}} \approx 0.6 \mathrm{ps}$ and $\beta=0.5$ yields an average decay time $\langle\tau\rangle$ of about $1.2 \mathrm{ps}$. We find the same time constant of $140 \mathrm{fs}$ for the delayed buildup of the polaron signal, followed by a decay characterized by an average decay time $\langle\tau\rangle$ of about $0.8 \mathrm{ps}$. The fact that the rise of the polaron population is correlated with a simultaneous decay of the exciton population is strong evidence that the polarons are created through the dissociation of singlet excitons. ${ }^{72}$

From the overview of the fit parameters in Figure 6b, we see that the time constants found in our fits stay rather constant over the entire spectral range of the individual transient species. Moreover, the fastest time constant $\tau_{1}$ is present over the entire probe spectral range. This implies that the chosen model describes the intrinsic dynamics properly. The variations of the $\tau_{\mathrm{SE}}$ decay time between the transient species can be attributed to different recombination processes for polarons and excitons as well as energy transfer processes between coils and aggregates suggested above and demonstrated in more detail below. The amplitudes for the ultrafast 140 fs component $\left(A_{1}\right)$ and the slower component $\left(A_{\mathrm{SE}}\right)$ have the same sign in the spectral region describing the singlet exciton absorption, that is, from 900 to $1150 \mathrm{~nm}$ and beyond. For shorter probe wavelengths, when the polaron absorption is probed, the amplitude $A_{1}$ for the $140 \mathrm{fs}$ component changes sign, as it no longer describes an absorption decay but rather the delayed rise of the polaron absorption. This evident correlation between singlet exciton decay and polaron rise further strengthens our interpretation. Similar kinetics are observed for neat P3HT, RRa-P3HT/poly-Si PHJ, and RRP3HT:Si-ncs BHJ, independent of pump polarization orientation. At $6 \mathrm{~ns}$, the GSB shows $5 \%$ of its initial signal magnitude that we assign to long-lived photoexcitations, which can readily be harvested in a solar cell. At this point we want to emphasize that the samples were optimized for ultrafast TA measurements and not for optimum solar cell performance, where reduced recombination can be achieved via various methods as detailed in the introduction and the conclusion sections.

For an absorbed number of photons of $2.6 \times 10^{9}$ per pulse at an excitation of $9 \mu \mathrm{J} / \mathrm{cm}^{2}$ at $518 \mathrm{~nm}$, we obtain an excited state areal density of $2.4 \times 10^{13} \mathrm{~cm}^{-2}$ (Table 3, see details in Supporting Information). The polaron cross section of $(3.4 \pm 2) \times$ $10^{-16} \mathrm{~cm}^{2}$, the polaron molar extinction coefficient of 
Table 3. Parameters of the P3HT/Si Heterojunction with $9 \mu \mathrm{J} / \mathrm{cm}^{2}$ Excitation at $518 \mathrm{~nm}$

\begin{tabular}{ll}
\multicolumn{1}{c}{ parameter } & \\
\hline absorbed photons & $2.6 \times 10^{9} /$ pulse \\
areal density of excitations $\left(\mathrm{cm}^{-2}\right)$ & $2.4 \times 10^{13}$ \\
polaron cross section $\left(\mathrm{cm}^{2}\right)$ & $(3.4 \pm 2) \times 10^{-16}$ \\
initial charge density $\left(\mathrm{cm}^{-3}\right)$ & $6 \times 10^{18}$ \\
\hline
\end{tabular}

Table 4. Initial Quantum Yields of Charges in Neat P3HT and aggRR-P3HT/poly-Si PHJ

\begin{tabular}{lrccl}
\multicolumn{1}{c}{ Sample } & ${\mathrm{P} 3 \mathrm{HT}^{+}}^{+}$ & $\mathrm{P}^{2} \mathrm{HT}^{-}$ & $\mathrm{P} 3 \mathrm{HT}+\mathrm{E}$ & $\mathrm{Si}^{-}$ \\
$\operatorname{aggP3HT}$ & 0.17 & 0.17 & 0.83 & 0 \\
$\operatorname{aggP3HT} / \mathrm{Si}$ & 0.38 & 0.13 & 0.62 & 0.25 \\
\hline
\end{tabular}

$(4 \pm 1) \times 10^{4} \mathrm{~L} \mathrm{~mol}^{-1} \mathrm{~cm}^{-1}$, and the initial charge density of $6 \times 10^{18} \mathrm{~cm}^{-3}$ obtained from our measurement series are similar to previous investigations of RR-P3HT:PCBM BHJ at comparable excitation fluence. ${ }^{16,30,73}$

With the interpretation that photoexcitation generates excitons that subsequently decay into polarons, it is possible to estimate the maximum yield of polarons formed initially by considering the relative magnitudes of the TA signals at $300 \mathrm{fs}$ given in Figure 5. Details of the calculations can be found in the Supporting Information. While we estimate a maximum quantum yield of $17 \%$ for the formation of the $\mathrm{P}_{3} \mathrm{HT}^{+}$polaron in a neat film of aggRR-P3HT, this value raises to $38 \%$ in the planar heterojunction (aggRR-P3HT/poly-Si PHJ) in combination with a maximum $\mathrm{Si}^{-}$yield of $25 \%$ (Table 4 ). We note that in the heterojunction device, the P3HT layer covering the silicon has a film thickness of only $40 \mathrm{~nm}$ so that excitons are created close to the donor-acceptor interface. The significant enhancement of exciton dissociation in the presence of silicon implies that silicon performs very well as an electron accepting material. The charge yield points to efficient electron transfer and a spatial exciton delocalization in ordered P3HT of about $10 \mathrm{~nm}$, in agreement with previous investigations. ${ }^{74,75}$

The obtained polaron yields are similar to RR-P3HT:PCBM studied previously, ${ }^{29}$ which raises the hope for aggRR-P3HT/Si heterojunctions to achieve comparable power conversion efficiencies as existing and even commercially available thin film solar cells based on RR-P3HT:PCBM BHJs. ${ }^{32}$

3.5. Charge Recombination Processes. So far we have substantiated the discussion of the yield and the time scale of the exciton dissociation into polarons. Now we address the issue of whether the positive and negative polarons formed are still Coulomb-bound as a geminate pair or whether they are free charge carriers. This question is crucial for the efficient operation of photovoltaic devices. There is no obvious reason why the TA spectra of bound polarons should be very different from those of free polarons. We are therefore not able to distinguish between bound and free polarons on the basis of the TA spectra. However, it is possible to distinguish the two species by considering their recombination kinetics. We expect a pair of geminately bound positive and negative polarons to recombine (radiatively or nonradiatively) with each other, i.e., monomolecularly. Their decay should therefore not be affected by the overall number density of bound polaron pairs that are formed upon photoexcitation. Further, the number density of bound polaron pairs formed should not impact on the singlet exciton population, thus leaving the decay kinetics of the singlet exciton absorption or the GSB unaltered. In contrast, if exciton dissociation results in the generation of free, i.e., nongeminate, positive and negative polarons, then these charges can only recombine when they meet each other, i.e., by bimolecular charge-charge annihilation. The probability of meeting the oppositely charged polaron thereby increases with the number density of polarons formed. ${ }^{16,73}$ Further, singlet excitons can recombine through quenching by free polarons, and the probability for this exciton-charge annihilation also increases with the number density of free polarons. ${ }^{16,76,77}$ Thus, in summary, for geminately bound polarons we expect the decay transients of polaron absorption, singlet exciton absorption, and GSB to be independent of excitation fluence, while we expect accelerated decays with increasing excitation fluence for free polarons generated upon singlet exciton dissociation.

To study the role of delocalization and the nature of the recombination processes in $\mathrm{P} 3 \mathrm{HT} / \mathrm{Si}$ heterojunctions, we record Vis-NIR TA spectra up to 2 ns delay with increased excitation fluences from 4 to $60 \mu \mathrm{J} / \mathrm{cm}^{2}$. The absorption signals of the corresponding relevant transient species in aggRR-P3HT/polySi PHJ (Figure 7a), RRa-P3HT/poly-Si PHJ (Figure 7b), and RR-P3HT:Si-ncs BHJ (Figure S8) are normalized against their individual initial maximum. This allows the study of the recombination rate as a function of excitation fluence. For the RRa$\mathrm{P} 3 \mathrm{HT} /$ poly-Si PHJ sample we find the decay kinetics to be independent of excitation fluence. Consequently, the polarons formed in a PHJ of silicon with RRa-P3HT are predominantly Coulomb-bound. In contrast, we find enhanced decay rates with increasing excitation fluence for aggRR-P3HT/poly-Si PHJ, suggesting the predominant formation of free polarons. The same observation is made for RR-P3HT:Si-ncs BHJs (Figure S8). In Figure 7a the enhanced decay rate leads to an apparent shift of the maximum of polaron absorption to earlier times, covering the delayed polaron rise.

Consequently, in the aggRR-P3HT/poly-Si PHJ and the RRP3HT:Si-ncs BHJ photoinduced ultrafast generation of free charges is obtained, which opens the route for efficient charge extraction from the active layer in the hybrid devices. In fact, it was recently shown that the competition between extraction and bimolecular recombination of mobile charges determines the dependence of the photocurrent on the applied bias and therefore the fill factor in RR-P3HT:PCBM BHJ devices. ${ }^{78,79}$

It is worthwhile to briefly reflect on these results. For the RRa$\mathrm{P} 3 \mathrm{HT} /$ poly-Si PHJ, photoexcitation near the hybrid interface results in excitons that are mostly localized on coiled chains. An electron is then transferred to the poly-Si with a time constant of $140 \mathrm{fs}$, leaving behind a $\mathrm{P}_{3} \mathrm{HT}^{+}$cation, i.e., a positive polaron. In silicon, the electron can be expected to be well delocalized because of the high dielectric screening. However, on the coiled P3HT chain, the conjugation length is low and the energetic disorder is high. As a result, the positive polaron is localized in the sense that its coherence length and its mobility are low. It seems that the presence of a more "pointlike" and moreover "immobile" positive charge on the $\mathrm{P} 3 \mathrm{HT}$ prevents the formation of free polarons. In a certain way, the situation is comparable to that of a point charge in front of a metal that feels an attractive force. In contrast for the aggRR-P3HT/poly-Si PHJ, a significant fraction of the excitations are created on planar, aggregated chains that are characterized by a high conjugation length and low energetic disorder. After charge transfer, the delocalized electron in the silicon is thus interacting with a positive polaron that not only is 

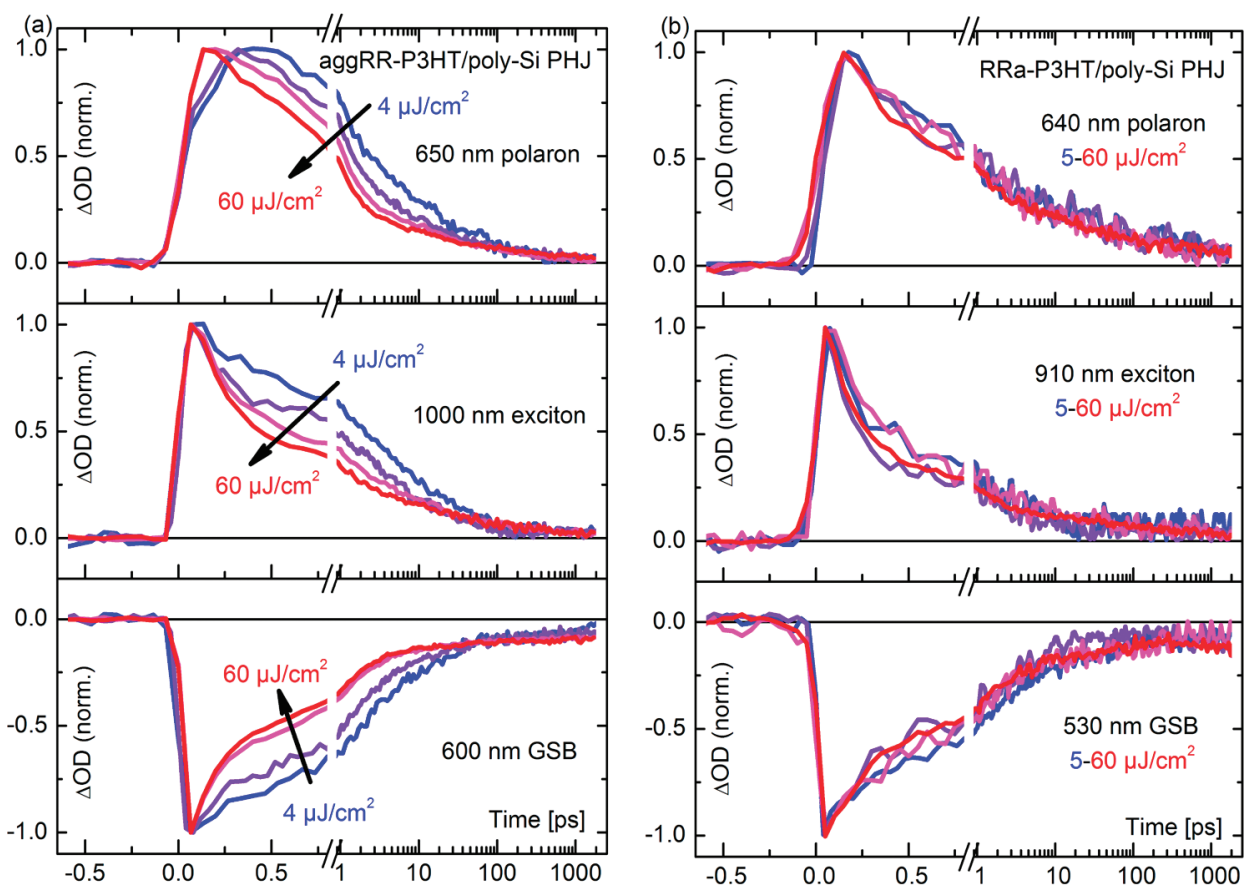

Figure 7. (a) Decay of TA signals for increased excitation fluences $\left(4,10,40\right.$, and $60 \mu \mathrm{J} / \mathrm{cm}^{2}$ at $518 \mathrm{~nm}$ ) reveals bimolecular nongeminate recombination in the case of aggRR-P3HT/poly-Si PHJ. (b) Decay of TA signals for increased excitation fluences $\left(5,20,40\right.$, and $60 \mu \mathrm{J} / \mathrm{cm}^{2}$ at $\left.518 \mathrm{~nm}\right)$ reveals monomolecular geminate recombination in the case of RRa-P3HT/poly-Si PHJ.

comparatively delocalized but also has a high initial mobility due to the low disorder. Such a polaron might move away from the hybrid interface, for example, by spectral diffusion to slightly longer conjugated segments, thereby overcoming the weak Coulomb attraction to the delocalized electron in the silicon and thus leading to the formation of a free pair of positive and negative charge. This finding is also supported by anisotropy measurements (Figure S10). It seems that a key issue in exciton dissociation is the delocalization, the dielectric screening, and the mobility of both the electron and the hole. Thus, a significant implication of the polymer structural order for hybrid and organic solar cells becomes evident and can be understood on a microscopic level.

Our interpretation of the results is based on the concept of singlet exciton dissociation into bound or free polaron pairs that we support by Figures 5 and 6 . From the excitation fluence of $4 \mu \mathrm{J} / \mathrm{cm}^{2}$, where bimolecular annihilation sets in, we derive a mean excitation spacing of more than $6 \mathrm{~nm}$ in aggRR-P3HT and RR$\mathrm{P} 3 \mathrm{HT}$, assuming an isotropic distribution of photoexcitations within the pump-probe volume. This finding reveals that despite the $6 \mathrm{~nm}(\sim 15$ thiophene repeating units) separation between the initial photoexcitations, the subsequent bimolecular interactions due to the generated mobile polarons can still take place pointing to high charge carrier mobility and rather spatially delocalized excitations, in agreement with other TA measurements for conjugated polymers, ${ }^{29,74}$ and supporting the degree of delocalization indicated from our quantum yield calculations mentioned above.

It was previously suggested that polarons in conjugated polymers may also be generated from higher excitonic states accessed by sequential excitation or by exciton-exciton annihilation. ${ }^{33,80-82}$ These delocalized "hot exciton" charge-transfer states are supposed to exhibit a higher dissociation probability via enhanced electronhole separation and charge mobility. ${ }^{75,83,84}$ These additional processes cannot, however, be the dominant polaron formation pathway in the present work for several reasons. First, the polaron formation happens with a $140 \mathrm{fs}$ time, which is too fast for bimolecular annihilation processes. ${ }^{16,77,82}$ Second, sequential excitations during the pump pulse come into play only at very high excitation fluences starting at $100-400 \mu \mathrm{J} / \mathrm{cm}^{2} .81,82$ Third, the polarons show the same intensity dependence as the excitons (Figure S9) and are thus generated from singlet excitons.

3.6. Variation of Excitation Wavelength.

3.6.1. Energy Transfer. We have seen that the conformation of the polymer chain has a major impact on the nature of the photogenerated charges. The number of excitations created on coiled chains or on aggregated chains is determined not only by the choice of $\mathrm{P} 3 \mathrm{HT}$ regioregularity and solvent but also by the choice of excitation wavelength. Figure 8 shows ultrafast UV-Vis TA spectra of neat RR-P3HT thin films at $60 \mathrm{fs,} 300$ fs, $13 \mathrm{ps}$, and $140 \mathrm{ps}$ with excitation at $450 \mathrm{~nm}$ (blue solid curve) and $600 \mathrm{~nm}$ (red solid curve). The spectra are normalized against the GSB so that signal changes solely inherent to the variation of the pump wavelength can be studied. The transient absorption spectra contain signatures of GSB $(425-625 \mathrm{~nm})$ and of polaron absorption $(>625 \mathrm{~nm})$. The inverted RR-P3HT film absorption spectrum (OD, green solid curve) is scaled to the RR-P3HT GSB peaks. The calculated difference spectra between the GSB and the inverted thin film absorption are shown as dashed curves for both excitation wavelengths (TA - OD, dashed). These difference spectra are then compared to the scaled absorption spectrum of RR-P3HT in dilute chloroform solution (OD, cyan solid line).

At $60 \mathrm{fs}$, the GSB of the RR-P3HT film is significantly broader when excited at $450 \mathrm{~nm}$ than for excitation at $600 \mathrm{~nm}$. Immediately after excitation with $600 \mathrm{~nm}$, the GSB of the film lacks the spectral components equivalent to the absorption spectrum in dilute chloroform solution. In dilute chloroform solution, polymers form coils as the chains curl up. Thus, at $60 \mathrm{fs}$ after excitation, only planar 


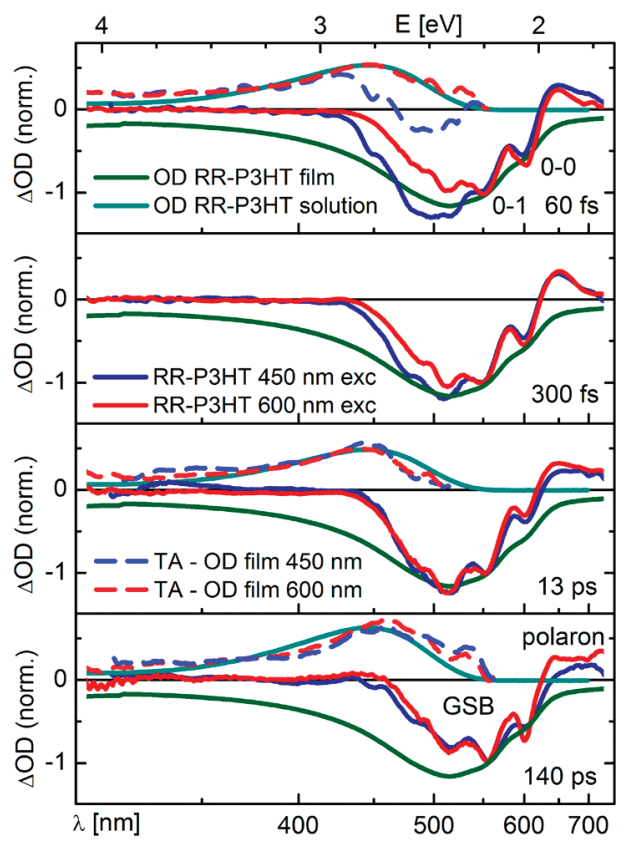

Figure 8. UV-Vis TA spectra of RR-P3HT thin film at $60 \mathrm{fs}, 300 \mathrm{fs}$, $13 \mathrm{ps}$, and $140 \mathrm{ps}$ with excitation at $450 \mathrm{~nm}$ (solid blue curve) and at $600 \mathrm{~nm}$ (solid red curve). The inverted absorption spectrum of RRP3HT thin film (OD, green solid curve) is scaled to the RR-P3HT GSB peaks to extract the differences (TA - OD, corresponding dashed curves) between the transient spectra and the film absorption, which are compared to the absorption spectrum of RR-P3HT in dilute chloroform solution (OD, cyan solid curve).

aggregated chains are excited by light with $600 \mathrm{~nm}$, while both coiled and aggregated chains are excited by light with $450 \mathrm{~nm}$. This distinct difference between the spectra obtained for excitation with $450 \mathrm{~nm}$ and with $600 \mathrm{~nm}$ stays visible up to $13 \mathrm{ps}$. At $13 \mathrm{ps}$, the film GSB coincides for both pump wavelengths. The difference between the GSB at 13 ps and the inverted film absorption spectrum corresponds to the absorption spectrum of P3HT coils except for some weak low energy tail around $480 \mathrm{~nm}$.

At 140 ps after excitation, the GSB shows even clearer vibrational structure and the difference between GSB and inverted film absorption spectrum reveals spectral parts missing in the GSB which are even beyond the coil absorption spectrum. This indicates further slow energy migration within the aggregates toward more planar and extended conjugated segments, e.g., via torsional relaxation or excitation energy transfer (EET) ${ }^{85,86}$ Thus, the GSB beyond 13 ps reveals the absorption spectrum due to RR-P3HT aggregates only, which is taken from the 140 ps case for Figure 9. It can be seen that aggregate absorption ranges from 460 to $625 \mathrm{~nm}$.

For excitation at $600 \mathrm{~nm}$, the GSB at $60 \mathrm{fs}$ and the GSB at $140 \mathrm{ps}$ are identical except for the changes in the $0-0$ peak intensity already discussed in the context of Figure 5. In contrast, for excitation at $450 \mathrm{~nm}$, the GSB loses the higher energy contributions that are attributed to coiled chains. We attribute this behavior to an energy transfer from unordered (coils) to ordered domains (aggregates, planar segments) in RR-P3HT films, as also indicated in the TA measurements of the PHJs and the $\mathrm{BHJ}$ above. Parts a and b of Figure 3 show that the PL of RR-P3HT in dilute solution (coils) overlaps with the absorption spectrum of RR-P3HT thin films, which is a prerequisite for efficient electronic energy transfer. ${ }^{68,70}$ With excitation of the RR-P3HT film at

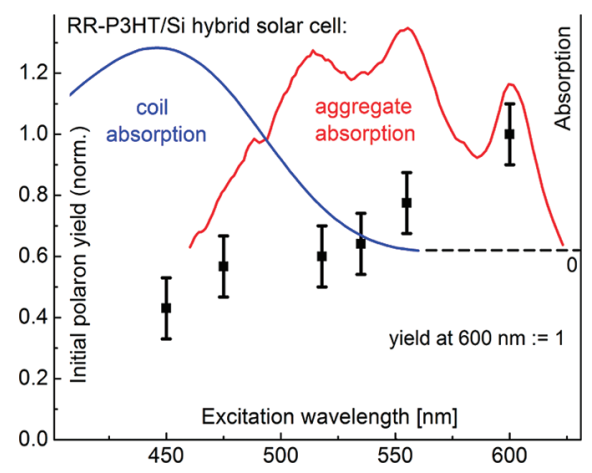

Figure 9. Dependence of initial polaron yield (black squares) on excitation wavelength for RR-P3HT/Si heterojunctions. Selective excitation of coiled (blue curve) vs aggregated (red curve) RR-P3HT domains reveals greater than a factor of 2 more efficient charge separation if exciting directly the aggregated RR-P3HT domains.

$450 \mathrm{~nm}$, both coils and aggregates are addressed; however, the coils undergo downhill energy transfer to the aggregated regions. An analysis of the ratio between the GSB intensity at $480 \mathrm{~nm}$ for excitation at 450 and at $600 \mathrm{~nm}$ reveals a forward $1 / e$ energy transfer time of about 3 ps (Figure S5, see more details in the Supporting Information). It is interesting to note that this is the same time constant as observed for the energy transfer from glassy phase to a planarized phase in poly $(9,9$ dioctylfluorene). ${ }^{37}$

3.6.2. Role of Excess Energy. Figure 8 allows us to distinguish the absorption range of coils and aggregates. For excitation wavelengths above $500 \mathrm{~nm}$ one predominantly addresses the aggregated RR-P3HT regions. For excitation above $550 \mathrm{~nm}$ one exclusively addresses the aggregated RR-P3HT regions. Furthermore, with excitation wavelengths below $500 \mathrm{~nm}$ one predominantly addresses the coiled RR-P3HT regions. We can therefore selectively excite ordered or disordered regions of P3HT films. Variation of the excitation wavelength thus provides an alternative approach to control whether coiled or aggregated chains are initially excited. We use this to further corroborate our results obtained on the morphology dependence of the polaron yield. Figure 9 shows the initial polaron yield at about $300 \mathrm{fs}$ as a function of the excitation wavelength at the hybrid heterojunction with the intermediately aggregated RR-P3HT. For comparison, the absorption spectra of coiled and of aggregated P3HT chains (taken as the GSB at 140 ps from Figure 8) are also shown.

For this experiment, the pump pulse was adjusted to central wavelengths of $450,475,518,535,555$, and $600 \mathrm{~nm}$ and pulse durations of about $15 \mathrm{fs}$ with the same experimental pump-probe conditions. For directly comparing the transient spectra of hybrid RR-P3HT/Si heterojunctions excited at different wavelengths, the transient spectra were scaled according to the individual initial P3HT GSB (internal standard). In this case, comparing the initial polaron absorption magnitudes of the various TA spectra can reveal changes in polaron yield solely inherent to the different excitation wavelengths while considering the same number of initial photoexcitations in the $\mathrm{P} 3 \mathrm{HT} / \mathrm{Si}$ heterojunction.

Figure 9 reveals an enhancement of more than a factor of 2 of initial polaron yield by increasing the excitation wavelength from 450 to $600 \mathrm{~nm}$. The trend was obtained independently on the device structure, i.e., for $\mathrm{PHJ}$ and $\mathrm{BHJ}$ geometries. The error bars result from multiple measurements of several hybrid RR-P3HT/ Si samples under the nominally same experimental conditions. 
The increase by a factor of 2 matches the enhancement of the polaron yield which was observed in Figure 5a,b by comparing highly aggregated aggRR-P3HT/poly-Si PHJ with RRa-P3HT/ poly-Si PHJ. In the latter case, P3HT coils were predominant. Our findings are an extension of previous TA investigations where the degree of aggregation was varied via thermal annealing. ${ }^{19}$ In conclusion, we record the same factor of 2 more efficient charge generation in aggregated polymer-based hybrid heterojunctions compared to the unaggregated version by two different and independent methods: (i) by using different P3HT configurations and therefore solely changing the structural order in the P3HT film (Figure 5) and (ii) by solely changing the excitation wavelength and therefore selectively exciting defined P3HT regions (Figure 9). If excess photon energy was necessary for the exciton dissociation process, we would expect a high initial polaron yield for excitation at $450 \mathrm{~nm}$ and a lower polaron yield for $600 \mathrm{~nm}$ excitation. The fact that we observe exactly the opposite tendency clarifies that excess photon energy is not required; however, structural order is essential. Whether this order enhances charge separation by increasing the initial charge carrier mobility or by improving the overall energetics or by both remains an intriguing question for further research.

\section{CONCLUSIONS AND IMPLICATIONS FOR HYBRID AND ORGANIC PHOTOVOLTAIC DEVICES}

We have comprehensively studied the nature of primary photoexcitations and their inherent dynamics in neat P3HT and in hybrid P3HT/Si thin films by ultrabroadband (UV-Vis-NIR) transient absorption (TA) spectroscopy with 40 fs time resolution and varied excitation wavelength. Hybrid heterojunctions with $30 \mathrm{~nm}$ thin polycrystalline Si layers or Si nanocrystals were processed with P3HT of varied polymer structural order and film geometry. The spatial and optical properties of planar and bulk heterojunctions show that $\mathrm{Si}$ does not change the P3HT structure and leads to a broad film absorption range from the UV to $1100 \mathrm{~nm}$ needed for efficient light-harvesting.

Scheme 1 summarizes the primary photoinduced processes in hybrid $\mathrm{P} 3 \mathrm{HT} / \mathrm{Si}$ thin film heterojunctions. In the TA experiments, we can identify the transient signatures of $\mathrm{P} 3 \mathrm{HT}$ polarons $(620-900 \mathrm{~nm})$ and singlet excitons $(900-1150 \mathrm{~nm})$. Our measurements reveal singlet excitons in $\mathrm{P} 3 \mathrm{HT}$ as primary photoexcitation with a subsequent ultrafast electron transfer from $\mathrm{P} 3 \mathrm{HT}$ to $\mathrm{Si}$ as inherent photovoltaic conversion process for all employed hybrid heterojunctions. The addition of Si to RR-P3HT or RRa-P3HT significantly enhances the polaron yield in the active layer

These experiments show that silicon is a particularly favorable electron acceptor because of the highly efficient charge delocalization. Moreover, the higher dielectric constant, compared to the state-of-the-art electron acceptor PCBM, allows for an improved screening of the electron, preventing back transfer. Besides these advantages compared to organic semiconductors, $\mathrm{Si}$ is abundantly available and offers the possibility of selective surface modifications and thus interface engineering.

In neat $\mathrm{P} 3 \mathrm{HT}$ and in $\mathrm{P} 3 \mathrm{HT} / \mathrm{Si}$ heterojunctions, we reveal a delayed polaron formation compared to singlet excitons, which appear within the experimental time resolution of $40 \mathrm{fs}$. The population of polarons has a maximum at about $300 \mathrm{fs}$ after excitation. Thus, charge generation is probed in real time, revealing a $140 \mathrm{fs}$ rise time for polarons, which is found to correlate with the initial $140 \mathrm{fs}$ decay of the singlet excitons in P3HT. We conclude that the
Scheme 1. Primary Photoinduced Processes in Hybrid P3HT/Si Thin Film Heterojunctions

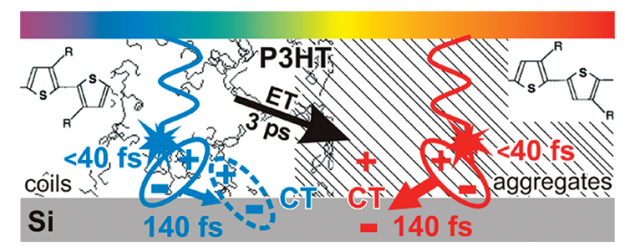

correlated decay of the exciton population and the rise of the polaron population indicate polaron formation via singlet exciton dissociation. In particular we stress that there is no significant polaron population immediately after excitation, i.e., after 40 fs. The ultrafast charge transfer (CT) process implies a strong exchange integral of the excited state orbitals of electron donor and acceptor.

This result demonstrates that the observation of ultrafast charge carrier generation is not in contradiction to the initial formation and subsequent dissociation of a singlet exciton, in contrast to recent suggestions made for the mechanism of charge carrier generation in the blend of the low band gap polymer PCDTBT with PCBM. ${ }^{31}$ Their argument essentially pertains to the fact that free charges can be observed as fast as $100 \mathrm{fs}$ after photoexcitation. Similar interpretations for a MDMO-PPV: PCBM blend have been made earlier. ${ }^{28}$ This issue, whether light absorption immediately creates free charge carriers or excitons, is central to the understanding and optimization of photoconversion in organic and hybrid solar cells. A problem in resolving this issue pertains to the experimental limits of time resolution and spectral range that are available for optical probing. Here we have developed a novel ultrabroadband gap-free TA spectroscopy setup with a time resolution of $40 \mathrm{fs}$ over the entire spectral range from 415 to $1150 \mathrm{~nm}$. This allows us to monitor both the decay of the primary excitation and its evolution into a charge pair state.

We have investigated the difference in the polaron formation process for RRa-P3HT, where only a small part of the film is composed of aggregates and for RR-P3HT containing an increased fraction of aggregates. TA measurements with varied excitation fluence clarify that in hybrid P3HT/Si heterojunctions with aggregated $\mathrm{P} 3 \mathrm{HT}$ exciton dissociation predominantly leads to free charge carriers, which can in principle be extracted as photocurrent. This is evident from the fact that in RR-P3HT/Si planar as well as in bulk heterojunctions we observe an increasing recombination rate of polarons with increasing excitation fluence, indicating bimolecular nongeminate recombination of charges outside the Coulombic capture radius. These recombination rates indicate that besides the primary photoconversion processes, the charge transport and extraction at the electrodes are crucial issues toward more efficient hybrid photovoltaic devices. Although we have already performed initial systematic studies on the reduction of Si dangling bond defects in the Si-ncs, ${ }^{58}$ which act as recombination centers, the charge transport and extraction need to be further addressed in future work. However, power conversion efficiencies of $1 \%$ have been shown recently with the first P3HT:Si-ncs blends. ${ }^{8}$ Combined with an optimized charge transport and extraction at the electrodes, their efficiency is expected to increase.

In contrast, for RRa-P3HT/Si, where there is initially a higher proportion of excitons on coiled $\mathrm{P} 3 \mathrm{HT}$ chains, the decay rate is 
independent of the excitation fluence, suggesting monomolecular geminate recombination of bound carriers, making it less suitable for photovoltaics from the photophysical perspective. Geminate recombination was recently also found for polymer-polymer blends and seems to be one of the main obstacles to be overcome for photovoltaic applications. ${ }^{77,87} \mathrm{We}$ attribute this difference between RRa- and RR-P3HT to a more localized hole in RRa$\mathrm{P} 3 \mathrm{HT}$ leading to localized charge carriers in RRa-P3HT/Si as opposed to highly delocalized charge carriers in RR-P3HT/Si revealing that high polymer structural order is a necessity for free charge generation in $\mathrm{P} 3 \mathrm{HT} / \mathrm{Si}$.

Quantitatively, we can demonstrate that using aggregated P3HT leads to a factor of 2 higher polaron yield compared to employing disordered $\mathrm{P} 3 \mathrm{HT}$ in photovoltaics, by two different and independent methods: (i) by using different P3HT configurations and therefore solely changing the structural order in the P3HT film and (ii) by solely changing the excitation wavelength and therefore selectively exciting defined $\mathrm{P} 3 \mathrm{HT}$ regions. Combined with results from a modified Franck-Condon analysis, we find that the polaron yield in $\mathrm{P} 3 \mathrm{HT} / \mathrm{Si}$ increases disproportionally with increasing degree of aggregation in P3HT. Moreover, we find that supplying excess energy does not assist the charge carrier separation, whereas our results indicate that ultrafast generation of free charges is more dependent on polymer structural order. We argue that the larger conjugation length, low energetic disorder, and the concomitant higher initial charge carrier mobility in the planar aggregated $\mathrm{P} 3 \mathrm{HT}$ compared to the short conjugation length in coiled P3HT favor the dissociation process into free charge carriers. Additionally, we observed that downhill energy transfer (ET) from coiled to aggregated chains takes place with a time constant of 3 ps.

For this reason, purely organic and hybrid photovoltaic devices using P3HT should employ highly aggregated P3HT. The loss of high-energy polymer absorption can be compensated by stacking heterojunctions in tandem or even multiple solar cells using conjugated polymers with different band gaps. ${ }^{88,89}$ Detailed investigations of the optimum Si-nc band gap for charge transfer remains for future research. The present ultrafast spectroscopic studies combined with ongoing P3HT/Si-ncs device optimization in terms of surface and defect properties of the $\mathrm{Si}$-ncs as well as of the film morphology raise the hope to realize efficient $\mathrm{P} 3 \mathrm{HT} / \mathrm{Si}$ photovoltaic devices.

\section{ASSOCIATED CONTENT}

S Supporting Information. Basic optical and structural characterization of P3HT: 3D AFM images of the samples and modified Franck-Condon analysis, description of the TA spectrometer, formulas of optical densities, cross sections and excitation densities, raw TA data for comparison, energy transfer from amorphous $\mathrm{P} 3 \mathrm{HT}$ to aggregated P3HT, quantum yields, $\mathrm{P} 3 \mathrm{HT}$ cation absorption spectrum in solution, TA measurements of P3HT in dilute solutions, bimolecular recombination, anisotropy measurements, exemplary $2 \mathrm{D}$ gap-free TA maps. This material is available free of charge via the Internet at http://pubs. acs.org.

\section{AUTHOR INFORMATION}

\section{Corresponding Author}

riedle@physik.uni-muenchen.de

\section{ACKNOWLEDGMENT}

The authors thank H. Bässler (Universität Bayreuth, Germany) for very fruitful discussions, P. Rupp and M. Bradler for experimental support, R. Tautz for help with the P3HT oxidation in solution, M. Algasinger and T. Antesberger for synthesizing the poly-Si, H. Wiggers for providing the Si-ncs, and J. Gmeiner for the synthesis of RRa-P3HT. D.H. is grateful to Studienstiftung des Deutschen Volkes. S.N. is thankful to the Karl-Max von Bauernfeind-Verein and the International Graduate School "Material Science for Complex Interfaces (CompInt)" of the Technische Universität München, Germany. C.S. and A.K. are thankful to the Graduiertenkolleg 1640 of the DFG. E.R. acknowledges funding from the SFB 749.

\section{REFERENCES}

(1) Thompson, B. C.; Fréchet, J. M. J. Angew. Chem., Int. Ed. 2008, $47,58-77$.

(2) Brabec, C. J.; Sariciftci, N. S.; Hummelen, J. C. Adv. Funct. Mater. 2001, 11, 15-26.

(3) Park, S. H.; Roy, A.; Beaupré, S.; Cho, S.; Coates, N.; Moon, J. S.; Moses, D.; Leclerc, M.; Lee, K.; Heeger, A. J. Nat. Photonics 2009, 3, 297-303.

(4) Gur, I.; Fromer, N. A.; Geier, M. L.; Alivisatos, A. P. Science 2005, 3, 462-465.

(5) Jabbour, G. E.; Doderer, D. Nat. Photonics 2010, 4, 604.

(6) Huynh, W. U.; Dittmer, J. J.; Alivisatos, A. P. Science 2002, $295,2425-2427$.

(7) Oosterhout, S. D.; Wienk, M. M.; van Bavel, S. S.; Thiedmann, R.; Koster, L. J. A.; Gilot, J.; Loos, J.; Schmidt, V.; Janssen, R. A. J. Nat. Mater. 2009, 8, 810-824.

(8) Liu, C.-Y.; Holman, Z. C.; Kortshagen, U. R. Nano Lett. 2009, 9, 449-452.

(9) Dietmueller, R.; Stegner, A. R.; Lechner, R.; Niesar, S.; Pereira, R. N.; Brandt, M. S.; Ebbers, A.; Trocha, M.; Wiggers, H.; Stutzmann, M. Appl. Phys. Lett. 2009, 94, 113301.

(10) Niesar, S.; Dietmueller, R.; Nesswetter, H.; Wiggers, H.; Stutzmann, M. Phys. Status Solidi A 2009, 206, 2775-2781.

(11) Beek, W. J. E.; Wienk, M. M.; Janssen, R. A. J. Adv. Funct. Mat. 2006, 16, 1112-1116.

(12) Briseno, A. L.; Holcombe, T. W.; Boukai, A. I.; Garnett, E. C.; Shelton, S. W.; Fréchet, J. M. C.; Yang, P. Nano Lett. 2010, 10, 334-340.

(13) Liu, J.; Kadnikova, E. N.; Liu, Y.; McGehee, M. D.; Fréchet, J. M. J. J. Am. Chem. Soc. 2004, 126, 9486-9487.

(14) Liu, J.; Tanaka, T.; Sivula, K.; Alivisatos, A. P.; Fréchet, J. M. J. J. Am. Chem. Soc. 2004, 126, 6550-6551.

(15) McDonald, S. A.; Konstantatos, G.; Zhang, S.; Cyr, P. W.; Klem, E. J. D.; Levina, L.; Sargent, E. H. Nat. Mater. 2005, 4, 138-142.

(16) Howard, I. A.; Mauer, R.; Meister, M.; Laquai, F. J. Am. Chem. Soc. 2010, 132, 14866-14876.

(17) Kim, Y.; Cook, S.; Tuladhar, S. M.; Choulis, S. A.; Nelson, J.; Durrant, J. R.; Bradley, D. D. C.; Giles, M.; McCulloch, I.; Ha, C.-S.; Ree, M. Nat. Mater. 2006, 5, 197-203.

(18) Peet, J.; Kim, J. Y.; Coates, N. E.; Ma, W. L.; Moses, D.; Heeger, A. J.; Bazan, G. C. Nat. Mater. 2007, 6, 497-500.

(19) Clarke, T.; Ballantyne, A. M.; Nelson, J.; Bradley, D. D. C.; Durrant, J. R. Adv. Funct. Mater. 2008, 18, 4029-4035.

(20) Spano, F. C. J. Chem. Phys. 2005, 122, 234701.

(21) Clark, J.; Silva, C.; Friend, R. H.; Spano, F. C Phys. Rev. Lett. 2007, 98, 206406.

(22) McCullough, R. D.; Tristram-Nagle, S.; Williams, S. P.; Lowe, R. D.; Jayaraman, M. J. Am. Chem. Soc. 1993, 115, 4910-4911.

(23) Chen, T.-A.; Wu, X; Rieke, R. D. J. Am. Chem. Soc. 1995, 117, 233-244.

(24) Sirringhaus, H.; Brown, P. J.; Friend, R. H.; Nielsen, M. M.; Bechgaard, K.; Langeveld-Voss, B. M. W.; Spiering, A. J. H.; Janssen, R. A. J.; Meijer, E. W.; Herwig, P.; de Leeuw, D. M. Nature 1999, 401, 685-688.

(25) Österbacka, R.; An, C. P.; Jiang, X. M.; Vardeny, Z. V. Science 2000, 287, 839-842. 
(26) Chang, J.-F.; Clark, J.; Zhao, N.; Sirringhaus, H.; Breiby, D. W.; Andreasen, J. W.; Nielsen, M. M.; Giles, M.; Heeney, M.; McCulloch, I. Phys. Rev. B 2006, 74, 115318.

(27) Mauer, R.; Kastler, M.; Laquai, F. Adv. Funct. Mater. 2010, 20, 2085-2092.

(28) Brabec, C. J.; Zerza, G.; Cerullo, G.; De Silvestri, S.; Luzzati, S.; Hummelen, J. C.; Sariciftci, S. Chem. Phys. Lett. 2001, 340, 232-236.

(29) Piris, J.; Dykstra, T. E.; Bakulin, A. A.; van Loosdrecht, P. H. M.; Knulst, W.; Trinh, M. T.; Schins, J. M.; Siebbeles, L. D. A. J. Phys. Chem. C 2009, 113, 14500-14506.

(30) Guo, J.; Ohkita, H.; Benten, H.; Ito, S. J. Am. Chem. Soc. 2010, $132,6154-6164$.

(31) Banerji, N.; Cowan, S.; Leclerc, M.; Vauthey, E.; Heeger, A. J. J. Am. Chem. Soc. 2010, 132, 17459-17470.

(32) Brabec, C. J.; Gowrisankar, S.; Halls, J. J. M.; Laird, D.; Jia, S.; Williams, S. P. Adv. Mater. 2010, 22, 3839-3856.

(33) Guo, J.; Ohkita, H.; Benten, H.; Ito, S. J. Am. Chem. Soc. 2009, 131, 16869-16880.

(34) Etzold, F.; Howard, I. A.; Mauer, R; Meister, M.; Kim, T.-D.; Lee, K.-S.; Baek, N. S.; Laquai, F. J. Am. Chem. Soc. 2011, 133, 9469-9479.

(35) Grancini, G.; Polli, D.; Fazzi, D.; Cabanillas-Gonzalez, J.; Cerullo, G.; Lanzani, G. J. Phys. Chem. Lett. 2011, 2, 1099.

(36) Yoshino, K.; Hayashi, S.; Sugimoto, R. Jpn. J. Appl. Phys. 1984, 23, L899.

(37) Khan, A. L. T.; Sreearunothai, P.; Herz, L. M.; Banach, M. J.; Köhler, A. Phys. Rev. B 2004, 69, 085201.

(38) Campbell, A. R.; Hodgkiss, J. M.; Westenhoff, S.; Howard, I. A.; Marsh, R. A.; McNeill, C. R.; Friend, R. H.; Greenham, N. C. Nano Lett. 2008, 8, 3942-3947.

(39) Scharsich, C; Lohwasser, R.; Asawapirom, U.; Scherf, U.; Thelakkat, M.; Köhler, A. Manuscript submitted, 2011.

(40) Knipping, J.; Wiggers, H.; Rellinghaus, B.; Roth, P.; Konjhodzic, D.; Meier, C. J. Nanosci. Nanotechnol. 2004, 4, 1039-1044.

(41) Germack, D. S.; Chan, C. K.; Kline, R. J.; Fischer, D. A.; Gundlach, D. J.; Toney, M. F.; Richter, L. J.; DeLongchamp, D. M. Macromolecules 2010, 43, 3828-3836.

(42) Scholz, M.; Gjukic, M.; Stutzmann, M. Appl. Phys. Lett. 2009, 94, 012108.

(43) Megerle, U.; Pugliesi, I.; Schriever, C.; Sailer, C. F.; Riedle, E. Appl. Phys. B: Lasers Opt. 2009, 96, 215-231.

(44) Wilhelm, T.; Piel, J.; Riedle, E. Opt. Lett. 1997, 22, 1494-1496.

(45) Riedle, E.; Beutter, M.; Lochbrunner, S.; Piel, J.; Schenkl, S.; Spörlein, S.; Zinth, W. Appl. Phys. B: Lasers Opt. 2000, 71, 457-465.

(46) Bradler, M.; Baum, P.; Riedle, E. Appl. Phys. B: Lasers Opt. 2009, 97, 561-574.

(47) Clark, J.; Chang, J.-F.; Spano, F. C.; Friend, R. H.; Silva, C. Appl. Phys. Lett. 2009, 94, 163306.

(48) Xu, B.; Holdcroft, S. Macromolecules 1993, 26, 4457-4460.

(49) Singh, R. K; Kumar, J.; Singh, R.; Kant, R.; Rastogi, R. C.; Chand, S.; Kumar, V. New J. Phys. 2006, 8, 112.

(50) Schueppel, R.; Schmidt, K.; Uhrich, C.; Schulze, K.; Wynands, D.; Brédas, J. L.; Brier, E.; Reinold, E.; Bu, H.-B.; Baeuerle, P.; Maennig, B.; Pfeiffer, M.; Leo, K. Phys. Rev. B 2008, 77, 085311.

(51) Jian, X. M.; Österbacka, R.; Korovyanko, O.; An, C. P.; Horovitz, B.; Janssen, R. A. J.; Vardeny, Z. V. Adv. Funct. Mater. 2002, 12, 587-597.

(52) van Hal, P. A.; Christiaans, M. P. T.; Wienk, M. M.; Kroon, J. M.; Janssen, R. A. J. J. Phys. Chem. B 1999, 103, 4352.

(53) Albert-Seifried, S; Friend, R. H. Appl. Phys. Lett. 2011, 98, 223304.

(54) Klessinger, M.; Michl, J. Excited States and Photochemistry of Organic Molecules; VCH Publishers, Inc.: New York, 1995; p 36.

(55) Köhler, A.; Bässler, H. Mater. Sci. Eng., R 2009, 66, 71-109.

(56) Ohkita, H.; Cook, S.; Astuti, Y.; Duffy, W.; Tierny, S.; Zhang, W.; Heeney, M.; McCulloch, I.; Nelson, J.; Bradley, D. D. C.; Durrant, J. R. J. Am. Chem. Soc. 2008, 130, 3030-3042.

(57) Tsoi, W. C.; Spencer, S. J.; Yang, L.; Ballantyne, A. M.; Nicholson, P. G.; Turnball, A.; Shard, A. G.; Murphy, C. E.; Bradley, D. D. C.; Nelson, J.; Kim, J.-S. Macromolecules 2011, 44, 2944-2952.
(58) Niesar, S.; Stegner, A. R.; Pereira, R. N.; Hoeb, M.; Wiggers, H.; Brandt, M. S.; Stutzmann, M. Appl. Phys. Lett. 2010, 96, 193112.

(59) Houston, P. L. Chemical Kinetics and Reaction Dynamics; Dover Publications, Inc.: Mineola, NY, 2001; pp 56-58.

(60) Middleton, C. T.; de La Harpe, K.; Su, C.; Law, Y. K.; CrespoHernández, C. E.; Kohler, B. Annu. Rev. Phys. Chem. 2009, 60, 217.

(61) Polli, D.; Altoè, P.; Weingart, O.; Spillane, K. M.; Manzoni, C.; Brida, D.; Tomasello, G.; Orlandi, G.; Kukura, P.; Mathies, R. A.; Garavelli, M.; Cerullo, G. Nature 2010, 467, 440.

(62) Chudoba, C.; Lutgen, S.; Jentzsch, T.; Riedle, E.; Woerner, M.; Elsaesser, T. Chem. Phys. Lett. 1995, 240, 35.

(63) Sobolewski, A. L.; Domcke, W.; Hättig, C. J. Phys. Chem. A 2006, 110, 6301.

(64) Petersson, J.; Eklund, M.; Davidsson, J.; Hammarström, L. J. Am. Chem. Soc. 2009, 131, 7940.

(65) Domcke, W.; Yarkony, D. R.; Köppel, H. Conical Intersections: Electronic Structure, Dynamics \& Spectroscopy (Advanced Series in Physical Chemistry); World Scientific Publishing Co., Inc.: Singapore, 2004.

(66) Köppel, H.; Domcke, W.; Yarkony, D. R. Conical Intersections: Theory, Computation and Experiments (Advanced Series in Physical Chemistry); World Scientific Publishing Co., Inc.: Singapore, 2011.

(67) Ruseckas, A.; Wood, P.; Samual, I. D. W.; Webster, G. R.; Mitchell, W. J.; Burn, P. L.; Sundström, V. Phys. Rev. B 2005, 72, 115214.

(68) Hwang, I.; Scholes, G. D. Chem. Mater. 2011, 23, 610-620.

(69) Lin, H.; Tian, Y.; Zapadka, K.; Persson, G.; Thomsson, D.; Mirzov, O.; Larsson, P.-O.; Widengren, J.; Scheblykin, I. G. Nano Lett. 2009, 9, 4456.

(70) Laquai, F.; Park, Y.-S.; Kim, J.-J.; Basché, T. Macromol. Rapid Commun. 2009, 30, 1203-1231.

(71) Movaghar, B.; Grünewald, M.; Ries, B.; Bässler, H.; Würtz, D. Phys. Rev. B 1986, 33, 5545-5554.

(72) Gulbinas, V.; Zaushitsyn, Y.; Sundström, V.; Hertel, D.; Bässler, H.; Yartsev, A. Phys. Rev. Lett. 2002, 89, 107401.

(73) Shuttle, C. G.; O’Regan, B.; Ballantyne, A. M.; Nelson, J.; Bradley, D. D. C.; Durrant, J. R. Phys. Rev. B 2008, 78, 113201.

(74) Dogariu, A.; Vacar, D.; Heeger, A. J. Phys. Rev. B 1998, $58,10218-10224$

(75) Köhler, A.; dos Santos, D. A.; Beljonne, D.; Shuai, Z.; Brédas, J,-L.; Kraus, A.; Müllen, K.; Friend, R. H. Nature 1998, 392, 903-906.

(76) Ferguson, A. J.; Kopidakis, N.; Shaheen, S. E.; Rumbles, G. J. Phys. Chem. C 2008, 112, 9865-9871.

(77) Howard, I. A.; Hodgkiss, J. M.; Zhang, X.; Kirov, K. R.; Bronstein, H. A.; Williams, C. K.; Friend, R. H.; Westenhoff, S.; Greenham, N. C. J. Am. Chem. Soc. 2010, 132, 328-335.

(78) Shuttle, C. G.; Hamilton, R.; O’Regan, B. C.; Nelson, J.; Durrant, J. R. Proc. Natl. Acad. Sci. U.S.A. 2010, 107, 16448-16452.

(79) Mauer, R.; Howard, I. A.; Laquai, F. J. Phys. Chem. Lett. 2010, $1,3500-3505$.

(80) Frolov, S. V.; Bao, Z.; Wohlgenannt, M.; Vardeny, Z. V. Phys. Rev. Lett. 2000, 85, 2196-2199.

(81) Stevens, M. A.; Silca, C.; Russell, D. M.; Friend, R. H. Phys. Rev. B 2001, 63, 165213 .

(82) Silva, C.; Dhoot, A. S.; Russell, D. M.; Stevens, M. A.; Arias, A. C.; MacKenzie, J. D.; Greenham, N. C.; Friend, R. H. Phys. Rev. B 2001, 64, 125211.

(83) Arkhipov, V. I.; Emelianova, E. V.; Bässler, H. Phys. Rev. Lett. $1999,82,1321-1324$.

(84) Zenz, C.; Lanzani, G.; Cerullo, G.; Graupner, W.; Leising, G.; Scherf, U.; DeSilvestri, S. Synth. Met. 2001, 116, 27-30.

(85) Parkinson, P.; Müller, C.; Stingelin, N.; Johnson, M. B.; Herz, L. M. J. Phys. Chem. Lett. 2010, 1, 2788-2792.

(86) Westenhoff, S.; Beenken, W. J. D.; Friend, R. H.; Greenham, N. C.; Yartsev, A.; Sundström, V. Phys. Rev. Lett. 2006, 97, 166804.

(87) Hodgkiss, J. M.; Campbell, A. R.; Marsh, R. A.; Rao, A.; AlbertSeifried, S.; Friend, R. H. Phys. Rev. Lett. 2010, 104, 177701.

(88) Kim, J. Y.; Lee, K.; Coates, N. E.; Moses, D.; Nguyen, T.-Q.; Dante, M.; Heeger, A. J. Science 2007, 317, 222-225.

(89) Gilot, J.; Wienk, M. M.; Janssen, R. A. J. Appl. Phys. Lett. 2007, 90, 143512. 